\title{
STOCHASTIC VOLATILITY: LIKELIHOOD INFERENCE AND COMPARISON WITH ARCH MODELS
}

\author{
SANGJOON KIM \\ Salomon Brothers Asia Limited, 5-2-20 Akasaka, Minato-ku, Tokyo 107, JAPAN \\ NEIL SHEPHARD \\ Nuffield College, Oxford University, Oxford OX1 1NF, UK \\ and \\ SiddhaRTHA CHIB \\ John M. Olin School of Business, Washington University, St Louis, MO 63130, USA
}

July 14, 1997

\begin{abstract}
In this paper, Markov chain Monte Carlo sampling methods are exploited to provide a unified, practical likelihood-based framework for the analysis of stochastic volatility models. A highly effective method is developed that samples all the unobserved volatilities at once using an approximating offset mixture model, followed by an importance reweighting procedure. This approach is compared with several alternative methods using real data. The paper also develops simulation-based methods for filtering, likelihood evaluation and model failure diagnostics. The issue of model choice using non-nested likelihood ratios and Bayes factors is also investigated. These methods are used to compare the fit of stochastic volatility and GARCH models. All the procedures are illustrated in detail.
\end{abstract}

(First version received December 1994)

Some key words: Bayes estimation, Bayes factors, Factor stochastic volatility, GARCH, Gibbs sampler, Heteroscedasticity, Maximum likelihood, Likelihood ratio, Markov chain Monte Carlo, Marginal likelihood, Quasi-maximum likelihood, Simulation, Stochastic volatility, Stock returns. 


\section{INTRODUCTION}

The variance of returns on assets tends to change over time. One way of modelling this feature of the data is to let the conditional variance be a function of the squares of previous observations and past variances. This leads to the autoregressive conditional heteroscedasticity (ARCH) based models developed by Engle (1982) and surveyed in Bollerslev, Engle, and Nelson (1994).

An alternative to the ARCH framework is a model in which the variance is specified to follow some latent stochastic process. Such models, referred to as stochastic volatility (SV) models, appear in the theoretical finance literature on option pricing (see, for example, Hull and White (1987) in their work generalizing the Black-Scholes option pricing formula to allow for stochastic volatility). Empirical versions of the SV model are typically formulated in discrete time. The canonical model in this class for regularly spaced data is:

$$
\begin{aligned}
y_{t} & =\beta e^{h_{t} / 2} \varepsilon_{t}, t \geq 1 \\
h_{t+1} & =\mu+\phi\left(h_{t}-\mu\right)+\sigma_{\eta} \eta_{t}, t \geq 2 \\
h_{1} & \sim \mathcal{N}\left(\mu, \frac{\sigma^{2}}{1-\phi^{2}}\right),
\end{aligned}
$$

where $y_{t}$ is the mean corrected return on holding the asset at time $t, h_{t}$ is the log volatility at time $t$ which is assumed to follow a stationary process $(|\phi|<1)$ with $h_{1}$ drawn from the stationary distribution, $\varepsilon_{t}$ and $\eta_{t}$ are uncorrelated standard normal white noise shocks and $\mathcal{N}(.,$.$) is the$ normal distribution. The parameter $\beta$ or $\exp (\mu / 2)$ plays the role of the constant scaling factor and can be thought of as the modal instantaneous volatility, $\phi$ as the persistence in the volatility, and $\sigma_{\eta}$ the volatility of the log-volatility. For identifiability reasons either $\beta$ must be set to one or $\mu$ to zero. We show later that the parameterization with $\beta$ equal to one in preferable and so we shall leave $\mu$ unrestricted when we estimate the model but report results for $\beta=\exp (\mu / 2)$ as this parameter has more economic interpretation.

This model has been used as an approximation to the stochastic volatility diffusion by Hull and White (1987) and Chesney and Scott (1989). Its basic econometric properties are discussed in Taylor (1986), the review papers by Taylor (1994), Shephard (1996) and Ghysels, Harvey, and Renault (1996) and the paper by Jacquier, Polson, and Rossi (1994). These papers also review the existing literature on the estimation of SV models.

In this paper we make advances in a number of different directions and provide the first complete Markov chain Monte Carlo simulation-based analysis of the SV model (1) that covers efficient methods for Bayesian inference, likelihood evaluation, computation of filtered volatility estimates, diagnostics for model failure, and computation of statistics for comparing non-nested volatility models. Our study reports on several interesting findings. We consider a very simple 
Bayesian method for estimating the SV model (based on one-at-a-time updating of the volatilities). This sampler is shown to quite inefficient from a simulation perspective. An improved (multi-move) method that relies on an offset mixture of normals approximation to a log-chisquare distribution coupled with a importance reweighting procedure is shown to be strikingly more effective. Additional refinements of the latter method are developed to reduce the number of blocks in the Markov chain sampling. We report on useful plots and diagnostics for detecting model failure in a dynamic (filtering) context. The paper also develops formal tools for comparing the basic SV and Gaussian and t-GARCH models. We find that the simple SV model typically fits the data as well as more heavily parameterized GARCH models. Finally, we consider a number of extensions of the SV model that can be fitted using our methodology.

The outline of this paper is as follows. Section 2 contains preliminaries. Section 3 details the new algorithms for fitting the SV model. Section 4 contains methods for simulation-based filtering, diagnostics and likelihood evaluations. The issue of comparing the SV and GARCH models is considered in Section 5. Section 6 provides extensions while Section 7 concludes. A description of software for fitting these models that is available through the internet is provided in Section 8. Two algorithms used in the paper are provided in the Appendix.

\section{PRELIMINARIES}

\subsection{Quasi-likelihood method}

A key feature of the basic SV model in (1) is that it can be transformed into a linear model by taking the logarithm of the squares of the observations

$$
\log y_{t}^{2}=h_{t}+\log \epsilon_{t}^{2}
$$

where $E\left(\log \epsilon_{t}^{2}\right)=-1.2704$ and $\operatorname{Var}\left(\log \epsilon_{t}^{2}\right)=4$.93. Harvey, Ruiz, and Shephard (1994) have employed Kalman filtering to estimate the parameters $\theta=\left(\phi, \sigma_{\eta}^{2}, \mu\right) \in(-1,1) \times \Re_{+} \times \Re$ by maximizing the quasi likelihood

$$
\log L_{Q}(y \mid \theta)=-\frac{n}{2} \log 2 \pi-\frac{1}{2} \sum_{t=1}^{n} \log F_{t}-\frac{1}{2} \sum_{t=1}^{n} v_{t}^{2} / F_{t},
$$

where $y=\left(y_{1}, \ldots, y_{n}\right), v_{t}$ is the one-step-ahead prediction error for the best linear estimator of $\log y_{t}^{2}$ and $F_{t}$ is the corresponding mean square error ${ }^{1}$. It turns out that this quasi-likelihood estimator is consistent and asymptotically normally distributed but is sub-optimal in finite samples because $\log \epsilon_{t}^{2}$ is poorly approximated by the normal distribution, as shown in Figure 1. As a consequence, the quasi-likelihood estimator under the assumption that $\log \epsilon_{t}^{2}$ is normal

\footnotetext{
${ }^{1}$ The Kalman filter algorithms for computing $v_{t}$ and $F_{t}$ are given in the Appendix.
} 
has poor small sample properties, even though the usual quasi-likelihood asymptotic theory is correct.
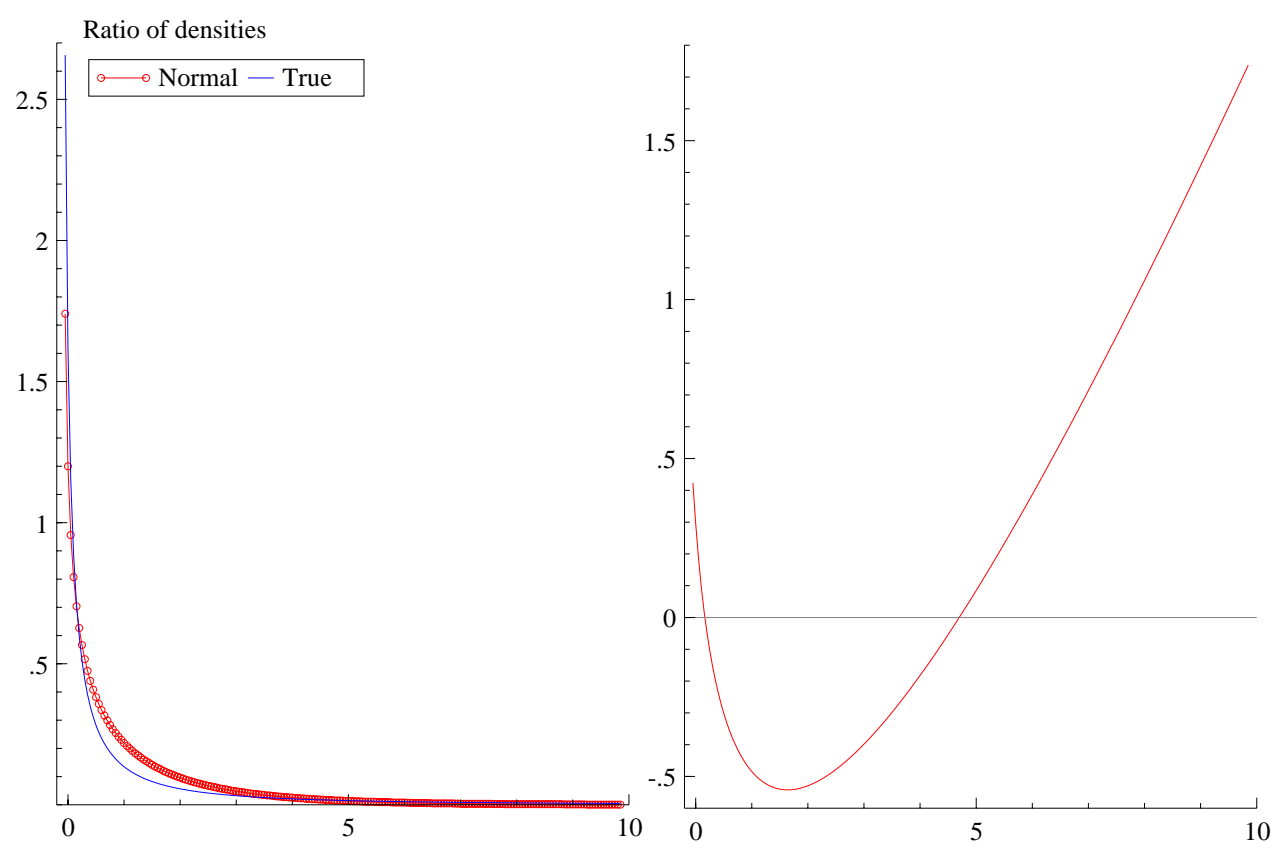

Figure 1: Log-Normal approximation to $\chi_{1}^{2}$ density. Left is the $\chi_{1}^{2}$ density and the log-normal approximation which is used in the quasi-likelihood approach. Right is the log of the ratio of the $\chi_{1}^{2}$ density to the approximation.

\subsection{Markov chain Monte Carlo}

An alternative, exact approach to inference in the SV model is based on Markov chain Monte Carlo (MCMC) methods, namely the Metropolis-Hastings and Gibbs sampling algorithms. These methods have had a widespread influence on the theory and practice of Bayesian inference. Early work on these methods appears in Metropolis, Rosenbluth, Rosenbluth, Teller, and Teller (1953), Hastings (1970), Ripley (1977) and Geman and Geman (1984) while some of the more recent developments, spurred by Tanner and Wong (1987) and Gelfand and Smith (1990), are included in Chib and Greenberg (1996), Gilks, Richardson, and Spiegelhalter (1996) and Tanner (1996, Ch. 6). Chib and Greenberg (1995) provide a detailed exposition of the Metropolis-Hastings algorithm and include a derivation of the algorithm from the logic of reversibility.

The idea behind MCMC methods is to produce variates from a given multivariate density (the posterior density in Bayesian applications) by repeatedly sampling a Markov chain whose invariant distribution is the target density of interest. There are typically many different ways 
of constructing a Markov chain with this property and one goal of this paper is to isolate those that are simulation-efficient in the context of SV models. In our problem, one key issue is that the likelihood function $f(y \mid \theta)=\int f(y \mid h, \theta) f(h \mid \theta) d h$ is intractable. This precludes the direct analysis of the posterior density $\pi(\theta \mid y)$ by MCMC methods. This problem can be overcome by focusing instead on the density $\pi(\theta, h \mid y)$, where $h=\left(h_{1}, \ldots, h_{n}\right)$ is the vector of $n$ latent volatilities. Markov chain Monte Carlo procedures can be developed to sample this density without computation of the likelihood function $f(y \mid \theta)$. It should be kept in mind that sample variates from a MCMC algorithm are a high-dimensional (correlated) sample from the target density of interest. These draws can be used as the basis for making inferences by appealing to suitable ergodic theorems for Markov chains. For example, posterior moments and marginal densities can be estimated (simulation consistently) by averaging the relevant function of interest over the sampled variates. The posterior mean of $\theta$ is simply estimated by the sample mean of the simulated $\theta$ values. These estimates can be made arbitrarily accurate by increasing the simulation sample size. The accuracy of the resulting estimates (the so called numerical standard error) can be assessed by standard time series methods that correct for the serial correlation in the draws. The serial correlation can be quite high for badly behaved algorithms.

\subsubsection{An initial Gibbs sampling algorithm for the SV model}

For the problem of simulating a multivariate density $\pi(\psi \mid y)$, the Gibbs sampler is defined by a blocking scheme $\psi=\left(\psi_{1}, \ldots, \psi_{d}\right)$ and the associated full conditional distributions $\psi_{i} \mid y, \psi_{\backslash i}$, where $\psi_{\backslash i}$ denotes $\psi$ excluding the block $\psi_{i}$. The algorithm proceeds by sampling each block from the full conditional distributions where the most recent values of the conditioning blocks are used in the simulation. One cycle of the algorithm is called a sweep or a scan. Under regularity conditions, as the sampler is repeatedly swept, the draws from the sampler converge to draws from the target density at a geometric rate. For the SV model the $\psi$ vector becomes $(h, \theta)$. To sample $\psi$ from the posterior density, one possibility (suggested by Jacquier, Polson, and Rossi (1994) and Shephard (1993)) is to update each of the elements of the $\psi$ vector one at a time.

1. Initialize $h$ and $\theta$.

2. Sample $h_{t}$ from $h_{t} \mid h_{\backslash t}, y, \theta, t=1, \ldots, n$.

3. Sample $\sigma_{\eta}^{2} \mid y, h, \phi, \mu, \beta$.

4. Sample $\phi \mid h, \mu, \beta, \sigma_{\eta}^{2}$.

5. Sample $\mu \mid h, \phi, \sigma_{\eta}^{2}$. 


\section{Goto 2.}

Cycling through 2 to 5 is a complete sweep of this (single move) sampler. The Gibbs sampler will require us to perform many thousands of sweeps to generate samples from $\theta, h \mid y$.

The most difficult part of this sampler is to effectively sample from $h_{t} \mid h_{\backslash t}, y_{t}, \theta$ as this operation has to be carried out $n$ times for each sweep. However,

$$
f\left(h_{t} \mid h_{\backslash t}, \theta, y\right) \propto f\left(h_{t} \mid h_{\backslash t}, \theta\right) f\left(y_{t} \mid h_{t}, \theta\right), \quad t=1, \ldots, n .
$$

We sample this density by developing a simple accept/reject procedure. ${ }^{2}$ Let $f_{N}(t \mid a, b)$ denote the normal density function with mean $a$ and variance $b$. It can be shown (ignoring end conditions to save space) that

$$
f\left(h_{t} \mid h_{\backslash t}, \theta\right)=f\left(h_{t} \mid h_{t-1}, h_{t+1}, \theta\right)=f_{N}\left(h_{t} \mid h_{t}^{*}, v^{2}\right),
$$

where

$$
h_{t}^{*}=\mu+\frac{\phi\left\{\left(h_{t-1}-\mu\right)+\left(h_{t+1}-\mu\right)\right\}}{\left(1+\phi^{2}\right)} \quad \text { and } \quad v^{2}=\frac{\sigma_{\eta}^{2}}{\left(1+\phi^{2}\right)} .
$$

Next we note that $\exp \left(-h_{t}\right)$ is a convex function and can be bounded by a function linear in $h_{t}$.

Let $\log f\left(y_{t} \mid h_{t}, \theta\right)=$ const $+\log f^{*}\left(y_{t}, h_{t}, \theta\right)$. Then

$$
\begin{aligned}
\log f^{*}\left(y_{t}, h_{t}, \theta\right) & =-\frac{1}{2} h_{t}-\frac{y_{t}^{2}}{2}\left\{\exp \left(-h_{t}\right)\right\} \\
& \leq-\frac{1}{2} h_{t}-\frac{y_{t}^{2}}{2}\left\{\exp \left(-h_{t}^{*}\right)\left(1+h_{t}^{*}\right)-h_{t} \exp \left(-h_{t}^{*}\right)\right\} \\
& =\log g^{*}\left(y_{t}, h_{t}, \theta, h_{t}^{*}\right) .
\end{aligned}
$$

Hence,

$$
f\left(h_{t} \mid h_{\backslash t}, \theta\right) f^{*}\left(y_{t}, h_{t}, \theta\right) \leq f_{N}\left(h_{t} \mid h_{t}^{*}, v^{2}\right) g^{*}\left(y_{t}, h_{t}, \theta\right) .
$$

The terms on the right hand side can be combined and shown to be proportional to $f_{N}\left(h_{t} \mid \mu_{t}, v^{2}\right)$ where

$$
\mu_{t}=h_{t}^{*}+\frac{v^{2}}{2}\left[y_{t}^{2} \exp \left(-h_{t}^{*}\right)-1\right] .
$$

\footnotetext{
${ }^{2}$ Five previous MCMC algorithms for simulating from $h_{t} \mid h_{t-1}, h_{t+1}, y_{t} ; \theta$ have been given in the literature by Shephard (1993), Jacquier, Polson, and Rossi (1994), Shephard and Kim (1994), Geweke (1994) and Shephard and Pitt (1997). The closest to our suggestion is Geweke (1994) who also bounded $\log f^{*}$, but by $-0.5 h_{t}$. This suffers from the property of having a high rejection rate for slightly unusual observations (for example, 0.9 for $\left.\left|y_{t}\right| / \beta \exp \left(h_{t} / 2\right)>3\right)$. Shephard and Pitt (1997), on the other hand, used a quadratic expansion of $\log f^{*}$ about $h_{t}^{*}$. This increases the generality of the procedure but it involves a Metropolis rejection step and so is more involved. Shephard (1993) approximated $f^{*}$ by a normal distribution with the same moments as $\log \epsilon_{t}^{2}$.

Geweke (1994) and Shephard and Kim (1994) independently suggested the use of the Gilks and Wild (1992) procedure for sampling from $\log$ concave densities such as $\log f\left(h_{t} \mid h_{\backslash t}, \theta, y\right)$. This is generalizable to non-logconcave densities using the Gilks, Best, and Tan (1995) sampler. Typically these routines need about 10 to 12 evaluations of $\log f\left(h_{t} \mid h_{\backslash t}, \theta, y\right)$ to draw a single random variable. Hence they are about 10 times less efficient than the simple accept/reject algorithm given above.

Jacquier, Polson, and Rossi (1994)'s Metropolis algorithm uses a very different approach. They approximate the density of $h_{t} \mid h_{\backslash t}$ and so use a non-Gaussian proposal based on $f^{*}$. Typically this procedure is considerably slower than the use of the Gilks and Wild (1992) methods suggested above.
} 
With these results, the accept-reject procedure (Ripley (1987)) to sample $h_{t}$ from $f\left(h_{t} \mid h_{\backslash t}, \theta, y\right)$ can now be implemented. First, propose a value $h_{t}$ from $f_{N}\left(h_{t} \mid \mu_{t}, v^{2}\right)$. Second, accept this value with probability $f^{*} / g^{*}$; if rejected return to the first step and make a new proposal. ${ }^{3}$

Sampling $\sigma_{\eta}^{2}$ and $\phi$ Sampling the $\sigma_{\eta}^{2}$ and $\phi$ one at a time is straightforward. If we assume a conjugate prior $\sigma_{\eta}^{2} \mid \phi, \mu \sim \mathcal{I} \mathcal{G}\left(\frac{\sigma_{r}}{2}, \frac{S_{\sigma}}{2}\right)$, then $\sigma_{\eta}^{2}$ is sampled from

$$
\sigma_{\eta}^{2} \mid y, h, \phi, \mu \sim \mathcal{I} \mathcal{G}\left\{\frac{n+\sigma_{r}}{2}, \frac{S_{\sigma}+\left(h_{1}-\mu\right)^{2}\left(1-\phi^{2}\right)+\sum_{t=1}^{n-1}\left(\left(h_{t+1}-\mu\right)-\phi\left(h_{t}-\mu\right)\right)^{2}}{2}\right\},
$$

where $\mathcal{I} \mathcal{G}$ denotes the inverse-gamma distribution. Throughout we set $\sigma_{r}=5$ and $S_{\sigma}=0.01 \times \sigma_{r}$.

For $\phi$, sampling from the full conditional density is also easy. Let $\phi=2 \phi^{*}-1$ where $\phi^{*}$ is distributed as Beta with parameters $\left(\phi^{(1)}, \phi^{(2)}\right)$. Hence, our prior on $\phi$ is

$$
\pi(\phi) \propto\left\{\frac{(1+\phi)}{2}\right\}^{\phi^{(1)}-1}\left\{\frac{(1-\phi)}{2}\right\}^{\phi^{(2)}-1}, \quad \phi^{(1)}, \phi^{(2)}>\frac{1}{2},
$$

and has support on the interval $(-1,1)$ with a prior mean of $\left\{2 \phi^{(1)} /\left(\phi^{(1)}+\phi^{(2)}\right)-1\right\}$. In our work we will select $\phi^{(1)}=20$ and $\phi^{(2)}=1.5$, implying a prior mean of 0.86 . Alternative priors could also be used. For example, the flat prior $\pi(\phi) \propto 1$ is attractive in that it leads to an analytically tractable full conditional density. But this prior can cause problems when the data are close to being non-stationary (Phillips (1991) and Schotman and Van Dijk (1991)). Chib and Greenberg (1994) and Marriott and Smith (1992) discuss other priors (restricted to the stationary region) for autoregressive models. We feel that it is important from a data-analytic view to impose stationarity in the SV model. Further, if $\phi=1$ then the $\mu$ terms cancel in (1) and so $\mu$ becomes unidentified from the data. The prior we select avoids these two problems rather well.

Under the specified prior, the full conditional density of $\phi$ is proportional to

$$
\pi(\phi) f\left(h \mid \mu, \phi, \sigma_{\eta}^{2}\right)
$$

where

$$
\log f\left(h \mid \mu, \phi, \sigma_{\eta}^{2}\right) \propto-\frac{\left(h_{1}-\mu\right)^{2}\left(1-\phi^{2}\right)}{2 \sigma_{\eta}^{2}}+\frac{1}{2} \log \left(1-\phi^{2}\right)-\sum_{t=1}^{n-1} \frac{\left\{\left(h_{t+1}-\mu\right)-\phi\left(h_{t}-\mu\right)\right\}^{2}}{2 \sigma_{\eta}^{2}},
$$

This function is concave in $\phi$ for all values of $\phi^{(1)}, \phi^{(2)}$. This means that $\phi$ can be sampled using an acceptance algorithm. Employ a first order Taylor expansion of the prior about

$$
\widehat{\phi}=\sum_{t=1}^{n-1}\left(h_{t+1}-\mu\right)\left(h_{t}-\mu\right) / \sum_{t=1}^{n-1}\left(h_{t}-\mu\right)^{2},
$$

\footnotetext{
${ }^{3}$ This proposal has an average acceptance rate of approximately $1-y_{t}^{2} \exp \left(-h_{t}^{*}\right) v_{t}^{2} /\left(4 \beta^{2}\right)$. A typical situation is where $v_{t}^{2}=0.01$. Usually $y_{t}^{2} \exp \left(-h_{t}^{*}\right) v_{t}^{2} / \beta^{2}$ will not be very large as $h_{t}^{*}$ is the smoothed log-volatility of $y_{t}$ and so reflects the variation in $y_{t}$. An extreme case is where $y_{t}^{2} \exp \left(-h_{t}^{*}\right) \sigma_{t}^{2} / \beta^{2}=100$, which leads to an average acceptance rate of approximately 0.75 . In our experience an average acceptance rate of over 0.995 seems usual for real financial datasets.
} 
and combine with $f\left(h \mid \mu, \phi, \sigma^{2}\right)$. The resulting density provides a good suggestion density. Alternatively, one can specialize the method of Chib and Greenberg (1994) (which is based on the Metropolis-Hastings algorithm). Given the current value $\phi^{(i-1)}$ at the $(i-1)$-st iteration, sample a proposal value $\phi^{*}$ from $N\left(\hat{\phi}, V_{\phi}\right)$ where $V_{\phi}=\sigma_{\eta}^{2}\left\{\sum_{t=1}^{n-1}\left(h_{t}-\mu\right)^{2}\right\}^{-1}$. Then, provided $\phi^{*}$ is in the stationary region, accept this proposal value as $\phi^{(i)}$ with probability $\exp \left\{g\left(\phi^{*}\right)-g\left(\phi^{(i-1)}\right)\right\}$ where

$$
g(\phi)=\log \pi(\phi)-\frac{\left(h_{1}-\mu\right)^{2}\left(1-\phi^{2}\right)}{2 \sigma_{\eta}^{2}}+\frac{1}{2} \log \left(1-\phi^{2}\right) .
$$

If the proposal value is rejected, set $\phi^{(i)}$ to equal $\phi^{(i-1)}$. Both these approaches can be used with alternative priors on $\phi$.

Sampling $\mu$ Suppose we work with a diffuse prior $^{4}$ on $\mu$, then $\mu$ is sampled from the full conditional density

$$
\mu \mid h, \phi, \sigma_{\eta}^{2} \sim \mathcal{N}\left(\hat{\mu}, \sigma_{\mu}^{2}\right)
$$

where

$$
\hat{\mu}=\sigma_{\mu}^{2}\left\{\frac{\left(1-\phi^{2}\right)}{\sigma_{\eta}^{2}} h_{1}+\frac{(1-\phi)}{\sigma_{\eta}^{2}} \sum_{t=1}^{n-1}\left(h_{t+1}-\phi h_{t}\right)\right\}
$$

and

$$
\sigma_{\mu}^{2}=\sigma_{\eta}^{2}\left\{(n-1)(1-\phi)^{2}+\left(1-\phi^{2}\right)\right\}^{-1}
$$

In our work we sample $\mu$ and record the value $\beta=\exp (\mu / 2)$.

Illustration To illustrate this algorithm we analyze the daily observations of weekday close exchange rates for the UK Sterling/US Dollar exchange rate from $1 / 10 / 81$ to $28 / 6 / 85$. The sample size is $n=946$. Later in the paper we will also use the corresponding series for the German Deutschemark (DM), Japanese Yen and Swiss Franc (SwizF), all against the US Dollar. This data set has been previously analysed using quasi-likelihood methods in Harvey, Ruiz, and Shephard (1994). The mean-corrected returns will be computed as

$$
y_{t}=100 \times\left\{\left(\log r_{t}-\log r_{t-1}\right)-\frac{1}{n} \sum_{i=1}^{n}\left(\log r_{i}-\log r_{i-1}\right)\right\},
$$

where $r_{t}$ denotes the exchange rate at time $t$. The MCMC sampler was initialized by setting all the $h_{t}=0$ and $\phi=0.95, \sigma_{\eta}^{2}=0.02$ and $\mu=0$. We iterated the algorithm on the log-volatilities for 1, 000 iterations and then the parameters and log-volatilities for 50,000 more iterations, before recording the draws from a subsequent 1,000,000 sweeps. The burn-in period is thus much larger than what is customary in the literature and is intended to ensure that the effect of the starting

\footnotetext{
${ }^{4}$ Occassionally, for technical reasons, we take a slightly informative prior such as $\mu \sim N(0,10)$. In this paper, this prior was used for the computation of Bayes factors.
} 
(a) phi|y against iteration

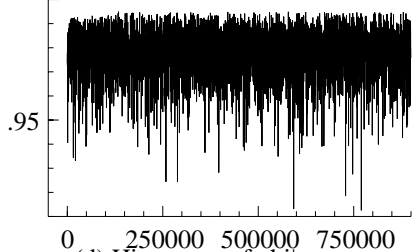

(d) Histogram of phily

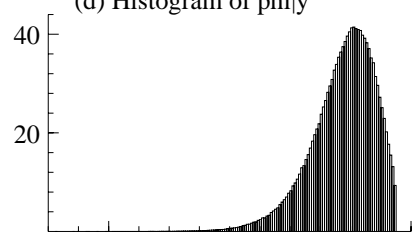

$9 \quad 95$

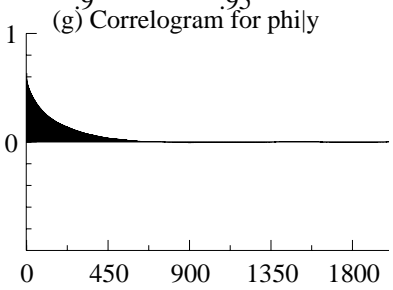

(b) sigma eta|y against iteration

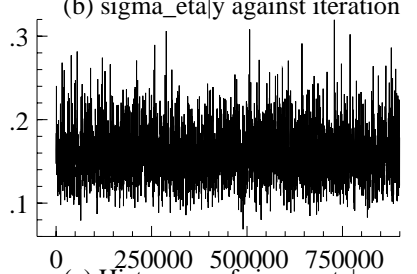

$0 \begin{array}{r}250000 \quad 500000 \quad 750000 \\ \text { (e) Histogram of sigma etaly }\end{array}$

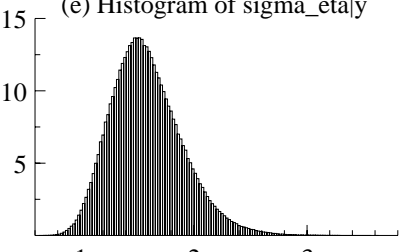
(h) Correlogram for sigma_eta|y

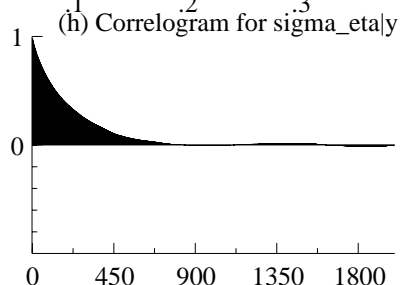

(c) beta|y against iteration
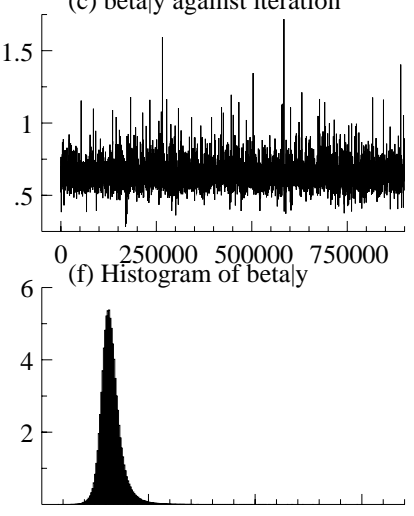

(i) Correlogram for beta|y
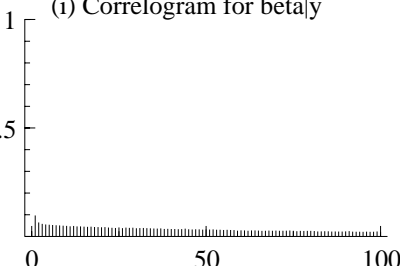

100

Figure 2: Single move Gibbs sampler for the Sterling series. Graphs (a)-(c): simulations against iteration. Graphs (d)-(f): histograms of marginal distribution. Graphs (g)-(i): corresponding correlograms for simulation. In total 1,000,000 iterations were drawn, discarding the first 50,000.

values becomes insignificant. As a result, there is likely to be no additional information from running multiple chains from dispersed starting values. The complete 1,000,000 iterations ${ }^{5}$ are graphed in Figure 2 and summarized in Table 1. ${ }^{6}$

The summary statistics of Table 1 report the simulation inefficiency factors of the sampler. These are estimated as the variance of the sample mean from the MCMC sampling scheme (the square of the numerical standard error) divided by the variance of the sample mean from a hypothetical sampler which draws independent random variables from the posterior (the variance divided by the number of iterations). We think that the simulation inefficiency statistic is a useful diagnostic (but by no means the only one) for measuring how well the chain mixes. The numerical standard error of the sample mean is estimated by time series methods (to account

\footnotetext{
${ }^{5}$ We have employed a 32 bit version of the modified Park and Miller (1988) uniform random number as the basis of all our random numbers. This has a period of $2^{31}-1$, which allows us to draw around 2.1 billion random numbers. In these experiments we are drawing approximately $n \times 2 \times 1.05$ random numbers per sweep of the sampler, where $5 \%$ is a very conservative estimate of the overall rejection rate. For this dataset this is 1984 draws per sweep. Given that we employ 1,000,000 sweeps, we are close, but not beyond, the period of our random number generator.

${ }^{6}$ Timings will be given for all the computations given in this paper. These are made using the authors $\mathrm{C}++$ code which has been linked to Ox. The single move algorithm is optimised to this special case and so is about as fast as it is possible to make it. The latter algorithms are much more general and so it is not completely fair to compare the computed time reported here to their times.
} 
for the serial correlation in the draws) as

$$
\widehat{R}_{B_{M}}=1+\frac{2 B_{M}}{B_{M}-1} \sum_{i=1}^{B_{M}} K\left(\frac{i}{B_{M}}\right) \widehat{\rho}(i),
$$

where $\widehat{\rho}(i)$ is an estimate of the autocorrelation at lag $i$ of the MCMC sampler, $B_{M}$ represents the bandwidth and $K$ the Parzen kernel (see, for example, Priestley (1981, Ch. 6)) given by

$$
\begin{aligned}
K(z) & =1-6 z^{2}+6 z^{3}, & & z \in\left[0, \frac{1}{2}\right] \\
& =2(1-z)^{3}, & & z \in\left[\frac{1}{2}, 1\right] \\
& =0, & & \text { elsewhere. }
\end{aligned}
$$

The correlogram (autocorrelation function) indicates important autocorrelations for $\phi$ and $\sigma_{\eta}$ at large lag lengths. If we require the Monte Carlo error in estimating the mean of the posterior to be no more than one percentage of the variation of the error due to the data, then this Gibbs sampler would have to be run for around 40,000 iterations. This seems a reasonably typical result: see Table 2 .

\begin{tabular}{lllrrrr}
\hline & Mean & MC S.E. & Inefficiency & \multicolumn{3}{c}{ Covariance \& Correlation } \\
$\phi \mid y$ & 0.97762 & 0.00013754 & 163.55 & 0.00011062 & -0.684 & 0.203 \\
$\sigma_{\eta} \mid y$ & 0.15820 & 0.00063273 & 386.80 & -0.00022570 & 0.00098303 & -0.129 \\
$\beta \mid y$ & 0.64884 & 0.00036464 & 12.764 & 0.00021196 & -0.00040183 & 0.0098569 \\
\hline Time & 5829.5 & 0.58295 & & & & \\
\hline
\end{tabular}

Table 1: Daily returns for Sterling: summaries of Figure 2. The Monte Carlo S.E. of simulation is computed using a bandwidth of 2,000, 4,000 and 2,000 respectively. Italics are correlations rather than covariances of the posterior. Computer time is seconds on a Pentium Pro/200. The other time is the number of seconds to perform 100 sweeps of the sampler.

\begin{tabular}{lllllll}
\hline & \multicolumn{2}{c}{$\phi \mid y$} & \multicolumn{2}{c}{$\sigma_{\eta} \mid y$} & \multicolumn{2}{c}{$\beta \mid y$} \\
Series & Mean & Inefficiency & Mean & Inefficiency & Mean & Inefficiency \\
DM & 0.96496 & 122.77 & 0.15906 & 292.81 & 0.65041 & 15.762 \\
Yen & 0.98010 & 313.03 & 0.12412 & 676.35 & 0.53597 & 14.192 \\
SwizF & 0.95294 & 145.48. & 0.20728 & 231.15 & 0.70693 & 13.700 \\
\hline
\end{tabular}

Table 2: Bandwidth was 2,000, 4,000 and 2,000, respectively for the parameters, for all series. In all cases 1,000,000 sweeps were used.

Parameterization An alternative to this sampler is to replace the draw for $\mu \mid h, \phi, \sigma_{\eta}^{2}$ with that resulting from the alternative parameterisation $\beta \mid y, h$. Such a move would be a mistake. Table 3 reports the inefficiency factor for this sampler using 1,000,000 draws of this sampler. There is a small deterioration in the sampler for $\phi \mid y$ and a very significant reduction in efficiency 
for $\beta \mid y$. The theoretical explanation for the inadequacies of the $\beta$ parameterization is provided by Pitt and Shephard (1998).

\begin{tabular}{lllllll}
\hline & \multicolumn{3}{c}{$\phi \mid y$} & \multicolumn{3}{c}{$\sigma_{\eta} \mid y$} \\
Series & Mean & Inefficiency & Mean & Inefficiency & Mean & Inefficiency \\
Sterling & 0.97793 & 465.30 & 0.15744 & 439.73 & 0.64280 & 5079.6 \\
\hline
\end{tabular}

Table 3: Bandwidth was 4,000, 4,000 and 15,000, respectively for the parameters. 1,000,000 sweeps were used.

Reason for slow convergence The intuition for the slow convergence reported in Table 1 is that the components of $h \mid y, \theta$ are highly correlated and in such cases sampling each component from the full conditional distribution produces little movement in the draws, and hence slowly decaying autocorrelations (Chib and Greenberg (1996)). For analytical results, one can think of the Gaussian equivalent of this problem. Under the Gaussian assumption and the linear approximation (2) and (1), the sampler in the simulation of $h$ from $h \mid y, \theta$ has an analytic convergence rate of ( Pitt and Shephard (1998, Theorem 1))

$$
4 \phi^{2} /\left\{1+\phi^{2}+\sigma_{\eta}^{2} / \operatorname{Var}\left(\log \varepsilon_{t}^{2}\right)\right\}^{2}
$$

where $\theta$ is taken as fixed at the expected values given in the results for the Sterling series. If $\operatorname{Var}\left(\log \varepsilon_{t}^{2}\right)$ is set equal to 4.93 , then this result implies a geometric convergence rate of $\rho_{A}=$ 0.9943 and an inefficiency factor of $\left(1+\rho_{A}\right) /\left(1-\rho_{A}\right)=350$ which is in the range reported in Table 1.

In order to improve the above sampler it is necessary to try to sample the log-volatilities in a different way. One method is to sample groups of consecutive log volatilities using a Metropolis algorithm. This is investigated in Shephard and Pitt (1997). In this paper we detail a more ambitious model specific approach. This approach is described next.

\section{OFFSET MIXTURE METHOD}

In this section we design an offset mixture of normals distribution (defined below) to accurately approximate the exact likelihood. This approximation helps in the production of an efficient (adapted Gibbs sampler) Monte Carlo procedure that allows us to sample all the log-volatilities at once. We then show how one can make the analysis exact by correcting for the (minor) approximation error by reweighting the posterior output. 


\subsection{The model}

Our approximating parametric model for the linear approximation (2) will be an offset mixture time series model

$$
y_{t}^{*}=h_{t}+z_{t}
$$

where $y_{t}^{*}=\log \left(y_{t}^{2}+c\right)$ and

$$
f\left(z_{t}\right)=\sum_{i=1}^{K} q_{i} f_{N}\left(z_{t} \mid m_{i}-1.2704, v_{i}^{2}\right)
$$

is a mixture of $K$ normal densities $f_{N}$ with component probabilities $q_{i}$, means $m_{i}-1.2704$, and variances $v_{i}^{2}$. The constants $\left\{q_{i}, m_{i}, v_{i}^{2}\right\}$ are selected to closely approximate the exact density of $\log \epsilon_{t}^{2}$. The "offset" $c$ was introduced into the SV literature by Fuller (1996, pp. 494-7) in order to robustify the QML estimator of the SV model to $y_{t}^{2}$ being very small. Throughout we will set $c=0.001$ (although it is possible to let $c$ depend on the actual value taken by $y_{t}^{2}$ ). It should be noted that the mixture density can also be written in terms of a component indicator variable $s_{t}$ such that

$$
\begin{aligned}
z_{t} \mid s_{t} & =i \sim \mathcal{N}\left(m_{i}-1.2704, v_{i}^{2}\right) \\
\operatorname{Pr}\left(s_{t}\right. & =i)=q_{i}
\end{aligned}
$$

This representation will be used below in the MCMC formulation.

We are now in a position to select $K$ and $\left\{m_{i}, q_{i}, v_{i}^{2}\right\}(i \leq K)$ to make the mixture approximation "sufficiently good". In our work, following for instance Titterington, Smith, and Makov (1985, p. 133), we matched the first four moments of $f_{\exp (Z)}(r)$ (the implied log-normal distribution) and $f\left(z_{t}\right)$ to those of a $\chi_{1}^{2}$ and $\log \chi_{1}^{2}$ random variable respectively, and required that the approximating densities lie within a small distance of the true density. This was carried out by using a non-linear least squares program to move the weights, means and variances around until the answers were satisfactory. It is worth noting that this nonlinear optimisation incurs only a one-time cost, as there are no model-dependent parameters involved. We found what we judged to be satisfactory answers by setting $K=7$. The implied weights, means and variances are given in Table 4, while the approximating and the true density are drawn in Figure 3. It would be easy to improve the fit by increasing the value of $K$, however further experiments that we have conducted suggest that increasing $K$ has little discernible effect on our main results.

\subsection{Mixture simulator}

In the MCMC context, mixture models are best estimated by exploiting the representation in (10). The general algorithm for state space models was suggested independently by Shephard 


\begin{tabular}{rrrr}
\hline \hline$\omega$ & $\operatorname{Pr}(\omega=i)$ & \multicolumn{1}{c}{$\mu_{i}$} & $\sigma_{i}^{2}$ \\
\hline 1 & 0.00730 & -10.12999 & 5.79596 \\
2 & 0.10556 & -3.97281 & 2.61369 \\
3 & 0.00002 & -8.56686 & 5.17950 \\
4 & 0.04395 & 2.77786 & 0.16735 \\
5 & 0.34001 & 0.61942 & 0.64009 \\
6 & 0.24566 & 1.79518 & 0.34023 \\
7 & 0.25750 & -1.08819 & 1.26261 \\
\hline
\end{tabular}

Table 4: Selection of the Mixing Distribution to be $\log \chi_{1}^{2}$.

(1994) and Carter and Kohn (1994). The posterior density of interest is $\pi\left(s, h, \phi, \sigma_{\eta}^{2}, \mu \mid y^{*}\right)$, where $s=\left(s_{1}, \ldots, s_{n}\right)$. In this case, both $h$ and $s$ can be sampled separately in one block and the sampler takes the form

1. Initialize $s, \phi, \sigma_{\eta}^{2}$ and $\mu$.

2. Sample $h$ from $h \mid y^{*}, s, \phi, \sigma_{\eta}^{2}, \mu$.

3. Sample $s$ from $s \mid y^{*}, h$.

4. Update $\phi, \sigma_{\eta}^{2}, \mu$ according to (6 ), (4) and (7).

\section{Goto 2.}

Note that we are using $y^{*}=\left\{\log \left(y_{1}^{2}+c\right), \ldots, \log \left(y_{T}^{2}+c\right)\right\}$ in the conditioning set above as a pointer to the mixture model. The vectors $y^{*}$ and $y$, of course, contain the same information.

The important improvement over the methods in section 2 is that it is now possible to efficiently sample from the highly multivariate Gaussian distribution $h \mid y^{*}, s, \phi, \sigma_{\eta}, \mu$ because $y^{*} \mid s, \phi, \sigma_{\eta}, \mu$ is a Gaussian time series which can be placed into the state-space form associated with the Kalman filter. The time series literature calls such models partially non-Gaussian or conditionally Gaussian. This particular model structure means we can sample from the entire $h \mid y^{*}, s, \phi, \sigma_{\eta}, \mu$ using the Gaussian simulation signal smoother detailed in the Appendix. As for the sampling of $s$ from $s \mid y^{*}, h$, this is done by independently sampling each $s_{t}$ using the probability mass function

$$
\operatorname{Pr}\left(s_{t}=i \mid y_{t}^{*}, h_{t}\right) \propto q_{i} f_{N}\left(y_{t}^{*} \mid h_{t}+m_{i}-1.2704, v_{i}^{2}\right), i \leq K
$$

The results from 750,000 sweeps of this mixture sampler are given in Table 5 and Figure 4. This sampler has less correlation than the single move sampler and suggests that generating 20,000 simulations from this sampler would probably be sufficient for inferential purposes. 

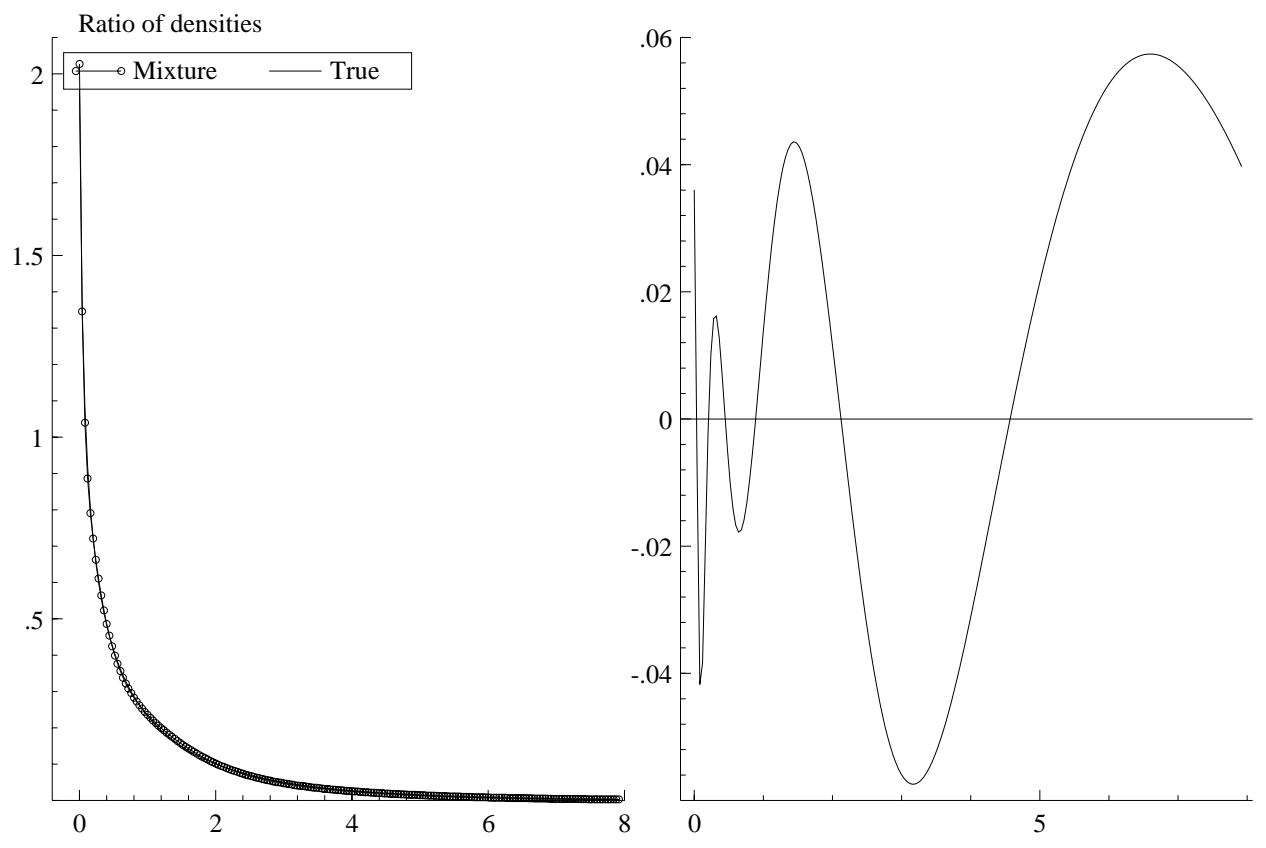

Figure 3: Mixture approximation to $\chi_{1}^{2}$ density. Left: $\chi_{1}^{2}$ density and mixture approximation. Right: the $\log$ of the ratio of the $\chi_{1}^{2}$ density to the mixture approximation.

\subsection{Integrating out the log-volatilities}

Although this mixture sampler improves the correlation behaviour of the simulations, the gain is not very big as there is a great deal of correlation between the volatilities and parameters. However, we can use the Gaussian structure of $y^{*} \mid s, \phi, \sigma_{\eta}^{2}$ to overcome this. We can sample the joint distribution $\pi\left(\phi, \sigma_{\eta}^{2}, h, \mu \mid y^{*}, s\right)$ by sampling $\left(\phi, \sigma_{n}^{2}\right)$ from $\pi\left(\phi, \sigma_{\eta}^{2} \mid y^{*}, s\right) \propto f\left(y^{*} \mid s, \phi, \sigma_{\eta}^{2}\right) \pi\left(\phi, \sigma_{\eta}^{2}\right)$, and then sampling $(h, \mu)$ from $\pi\left(h, \mu \mid y^{*}, s, \phi, \sigma_{\eta}^{2}\right)$. We are able to sample the former distribution because the density $f\left(y^{*} \mid s, \phi \sigma_{\eta}^{2}\right)$ can be evaluated using an augmented version of the Kalman

\begin{tabular}{lllrrrr}
\hline & Mean & MC S.E. & Inefficiency & \multicolumn{3}{c}{ Covariance \& Correlation } \\
$\phi \mid y$ & 0.97779 & $6.6811 \mathrm{e}-005$ & 29.776 & 0.00011093 & -0.690 & 0.203 \\
$\sigma_{\eta} \mid y$ & 0.15850 & 0.00046128 & 155.42 & -0.00023141 & 0.0010131 & -0.127 \\
$\beta \mid y$ & 0.64733 & 0.00024217 & 4.3264 & 0.00021441 & -0.00040659 & 0.010031 \\
\hline Time & 15374 & 2.0498 & & & & \\
\hline
\end{tabular}

Table 5: Daily returns for Sterling against Dollar. Summaries of Figure 2. The Monte Carlo S.E. of simulation is computed using a bandwidth of 2000, 2000 and 100 respectively. Italics are correlations rather than covariances of the posterior. Computer time is seconds on a Pentium Pro/200. The other time is the number of seconds to perform 100 complete passes of the sampler. 
(a) phi|y against iteration

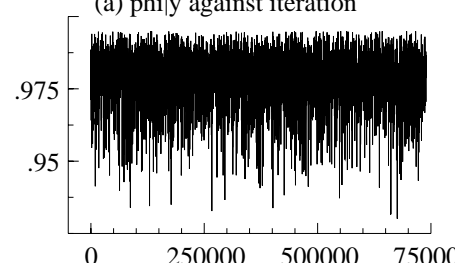

(d) Histogram of phily

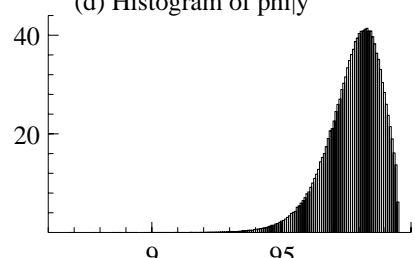

(g) Correlogram for phi|y

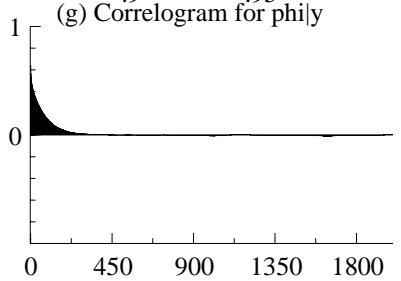

(b) sigma_eta|y against iteration

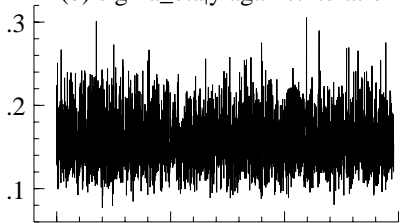

$0 \quad 250000 \quad 500000 \quad 750000$

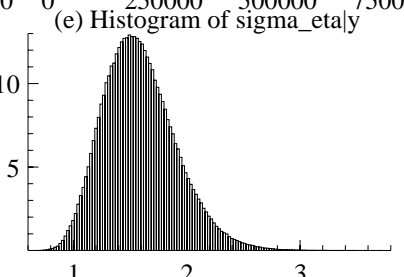

(h) Correlogram for sigma_eta|y

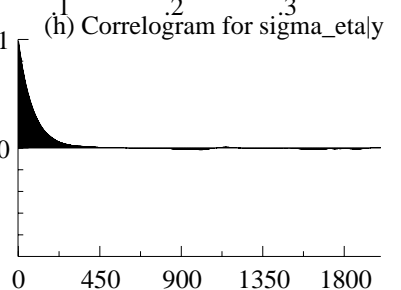

(c) betaly against iteration
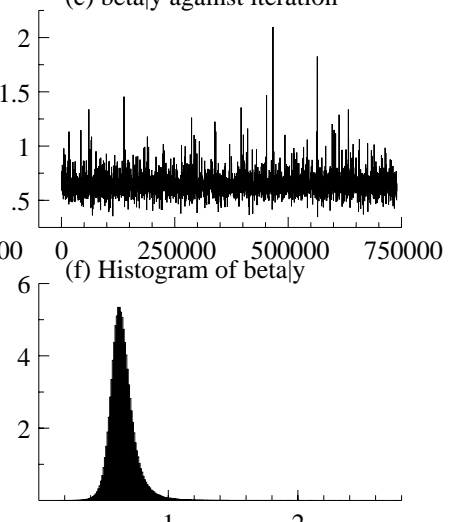

(i) Correlogram for beta|y

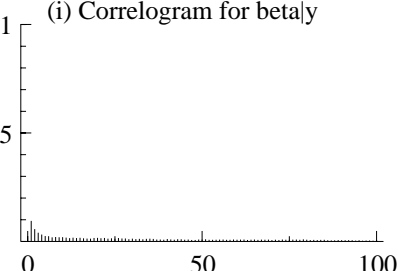

Figure 4: Mixture sampler for Sterling series. Graphs (a)-(c): simulations against iteration. Graphs (d)-(f): histograms of marginal distribution. Graphs (g)-(i): corresponding correlograms for simulation. In total 750,000 iterations were drawn, discarding the first 10,000.

filter (analytically integrating out $\mu$ and $h){ }^{7}$ Then, writing $\mu \mid y^{*}, s, \phi, \sigma_{\eta}^{2} \sim \mathcal{N}\left(\widetilde{\mu}, \sigma_{\widetilde{\mu}}^{2}\right)$ we have that

$$
\begin{aligned}
\pi\left(\phi, \sigma_{\eta}^{2} \mid y^{*}, s\right) & \propto \pi(\phi) \pi\left(\sigma_{\eta}^{2}\right) f\left(y^{*} \mid s, \phi, \sigma_{\eta}^{2}\right)=\pi(\phi) \pi\left(\sigma_{\eta}^{2}\right) \frac{f\left(y^{*} \mid s, \phi, \sigma_{\eta}^{2}, \mu=0\right) \pi(\mu=0)}{\pi\left(\mu=0 \mid y^{*}, s, \phi, \sigma_{\eta}^{2}\right)} \\
& \propto \pi(\phi) \pi\left(\sigma_{\eta}^{2}\right) \prod_{t=1}^{n} F_{t}^{-1 / 2} \exp \left(-\frac{1}{2} \sum_{t=1}^{n} v_{t}^{2} / F_{t}\right) \exp \left(\frac{1}{2 \sigma_{\widetilde{\mu}}^{2}} \widetilde{\mu}^{2}\right) \sigma_{\widetilde{\mu}},
\end{aligned}
$$

where $v_{t}$ is the one-step-ahead prediction error for the best mean square estimator of $y_{t}^{*}$, and $F_{t}$ is the corresponding mean square error. The quantities $v_{t}, F_{t}, \widetilde{\mu}, \sigma_{\widetilde{\mu}}^{2}$ are computed from the augmented Kalman filter provided in the Appendix, conditional on $s$.

This implies that we can sample from $\phi, \sigma_{\eta}^{2} \mid y^{*}, s$ directly by making the proposal $\left\{\phi^{(i)}, \sigma_{\eta}^{2(i)}\right\}$, given the current value $\left\{\phi^{(i-1)}, \sigma_{\eta}^{2(i-1)}\right\}$, by drawing from some density $g\left(\phi, \sigma_{\eta}^{2}\right)$ and then accepting them using the Metropolis-Hastings probability of move

$$
\min \left\{1, \frac{\pi\left(\phi^{(i)}, \sigma_{\eta}^{2(i)} \mid y^{*}, s\right)}{\pi\left(\phi^{(i-1)}, \sigma_{\eta}^{2(i-1)} \mid y^{*}, s\right)} \frac{g\left(\phi^{(i-1)}, \sigma_{\eta}^{2(i-1)}\right)}{g\left(\phi^{(i)}, \sigma_{\eta}^{2(i)}\right)}\right\} .
$$

If the proposal value is rejected, we then set $\left\{\phi^{(i)}, \sigma_{\eta}^{2(i)}\right\}=\left\{\phi^{(i-1)}, \sigma_{\eta}^{2(i-1)}\right\}$. We call this an 'integration sampler' as it integrates out the log-volatilities.

\footnotetext{
${ }^{7}$ Augmented Kalman filters and simulation smoothers are discussed in the Appendix.
} 
The structure of the integration sampler is then generically:

1. Initialize $\left(s, \phi, \sigma_{\eta}, \mu\right)$.

2. Sample $\left(\phi, \sigma_{\eta}^{2}\right)$ from $\pi\left(\phi, \sigma_{\eta}^{2} \mid y^{*}, s\right)$ using a Metropolis-Hastings suggestion based on $g\left(\sigma_{\eta}^{2}, \phi\right)$ accepting with probability (11).

3. Sample $h, \mu \mid y^{*}, s, \phi, \sigma_{\eta}^{2}$ using the augmented simulation smoother given in the Appendix.

4. Sample $s \mid y^{*}, h$ as in the previous algorithm.

5. Goto 2.

An important characteristic of this sampler is that the simulation smoother can jointly draw $h$ and $\mu$. The scheme allows a free choice of the proposal density $g\left(\phi, \sigma_{\eta}^{2}\right)$. We have employed a composite method which first draws 200 samples (discarding the first ten samples) from the posterior density $\pi\left(\phi, \sigma_{\eta}^{2} \mid y\right)$ using a Metropolis-Hastings sampler based on Gilks, Best, and Tan (1995) which only requires the coding of the function $y^{*} \mid s, \phi, \sigma_{\eta}^{2}$ and the prior. These 200 draws are used to estimate the posterior mean and covariance. The mean and twice the covariance are then used to form a Gaussian proposal density $g\left(\phi, \sigma_{\eta}^{2}\right)$ for the Metropolis-Hastings algorithm in (11). As an alternative, one could also use a multivariate Student t proposal distribution instead of the Gaussian. See Chib and Greenberg (1995) for further discussion on the issues involved in choosing a proposal density for the Metropolis-Hastings algorithm.

The output from the resulting sampler is reported in Figure 5 and Table 6 . These suggest that 2,000 samples from this generator would be sufficient for this problem. This result seems reasonably robust to the data set.

\begin{tabular}{lllrrrr}
\hline & Mean & MC S.E. & Inefficiency & \multicolumn{3}{c}{ Covariance \& Correlation } \\
$\phi \mid y$ & 0.97780 & $6.7031 \mathrm{e}-005$ & 9.9396 & 0.00011297 & -0.699 & 0.205 \\
$\sigma_{\eta} \mid y$ & 0.15832 & 0.00025965 & 16.160 & -0.00023990 & 0.0010426 & -0.131 \\
$\beta \mid y$ & 0.64767 & 0.00023753 & 1.4072 & 0.00021840 & -0.00042465 & 0.010020 \\
\hline Time & 8635.2 & 3.4541 & & & & \\
\hline
\end{tabular}

Table 6: Daily returns for Sterling against Dollar. Summaries of Figure 5. The Monte Carlo S.E. of simulation is computed using a bandwidth of 100, 100 and 100 respectively. Italics are correlations rather than covariances of the posterior. Computer time is seconds on a Pentium Pro/200. The other time is the number of seconds to perform 100 complete passes of the sampler.

\subsection{Reweighting}

The approach based on our (very accurate) offset mixture approximation provides a neat connection to conditionally Gaussian state space models and leads to elegant and efficient sampling 
(a) phily against iteration
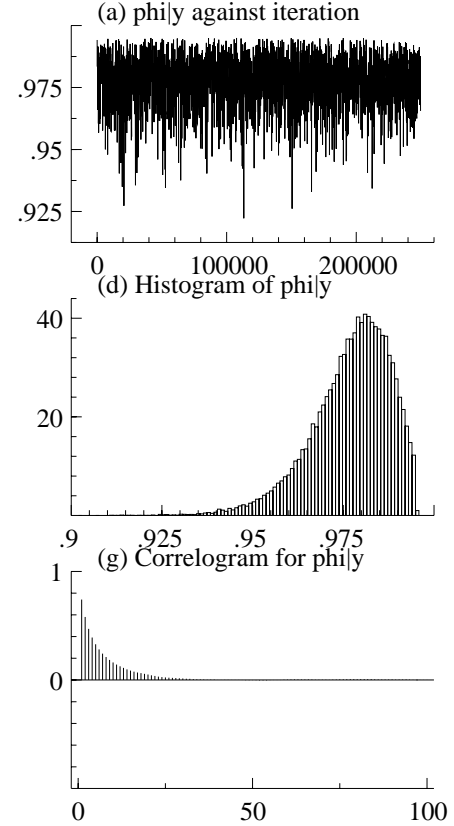

(b) sigma_eta|y against iteration

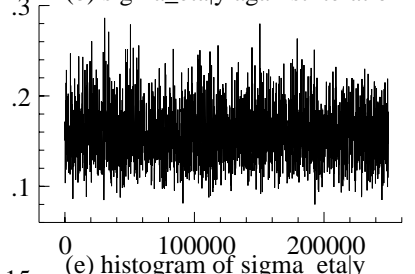

(e) histogram of sigma_eta|y

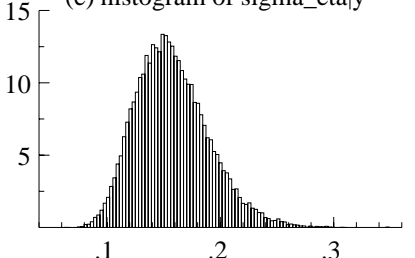

(h) $\stackrel{1}{\text { Correlogram }} .2$ for sigma_eta|y

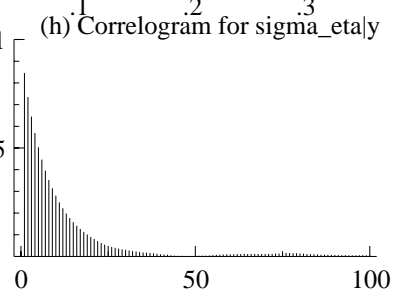

(c) beta|y against iteration
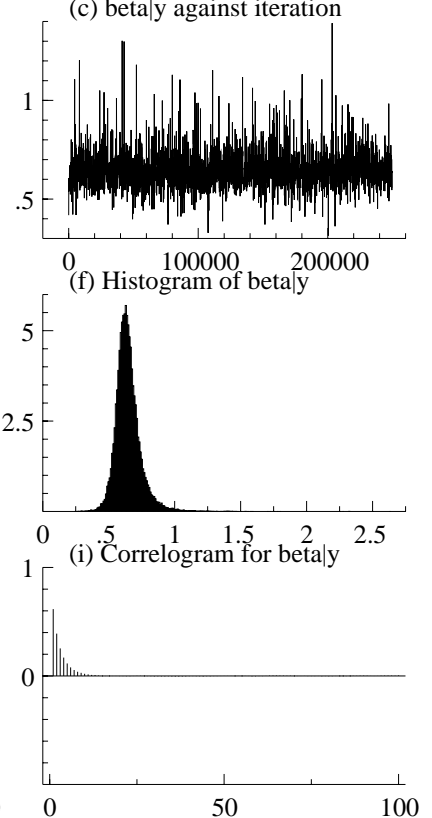

Figure 5: The integration sampler for Sterling series. Graphs (a)-(c): simulations against iteration. Graphs (d)-(f): histograms of marginal distribution. Graphs (g)-(i): corresponding correlograms for simulation. In total 250,000 iterations were drawn, discarding the first 250.

procedures, as shown above. We now show that it is possible to correct for the minor approximation error by appending a straightforward reweighting step at the conclusion of the above procedures. This step then provides a sample from the exact posterior density of the parameters and volatilities. The principle we describe is quite general and may be used in other simulation problems as well.

First write the mixture approximation as making draws from $k\left(\theta, h \mid y^{*}\right)$, and then define

$$
w(\theta, h)=\log f(\theta, h \mid y)-\log k(\theta, h \mid y)=\mathrm{const}+\log f(y \mid h)-\log k\left(y^{*} \mid h\right),
$$

where

$$
f(y \mid h)=\prod_{t=1}^{n} f_{N}\left\{y_{t} \mid 0, \exp \left(h_{t}\right)\right\}
$$

and

$$
k\left(y^{*} \mid h\right)=\prod_{t=1}^{n} \sum_{i=1}^{K} q_{i} f_{N}\left(y_{t}^{*} \mid h_{t}+m_{i}-1.2704, v_{i}^{2}\right) .
$$

Both these functions involve Gaussian densities and are straightforward to evaluate for any value of $h$. Then,

$$
\begin{aligned}
\mathrm{E} g(\theta) \mid y & =\int g(\theta) f(\theta \mid y) d \theta \\
& =\int g(\theta) \exp \{w(\theta, h)\} k\left(\theta, h \mid y^{*}\right) d \theta d h / \int \exp \{w(\theta, h)\} k\left(\theta, h \mid y^{*}\right) d \theta d h
\end{aligned}
$$


Thus we can estimate functionals of the posterior by reweighting the MCMC draws according to

$$
\mathrm{E} g \widehat{(\theta) \mid} y=\sum_{j} g\left(\theta^{j}\right) c^{j}
$$

where the weights are

$$
c^{j}=\exp \left\{w\left(\theta^{j}, h^{j}\right)\right\} / \sum_{i} \exp \left\{w\left(\theta^{i}, h^{i}\right)\right\}
$$

As the mixture approximation is very good, we would expect that the weights $c^{j}$ would have a small variance.

To see the dispersion of the weights, we recorded the weights from the sampler which generated Figure 5 and plotted the resulting log-weights in Figure 6. The log-weights are close to being normally distributed with a standard deviation of around one.

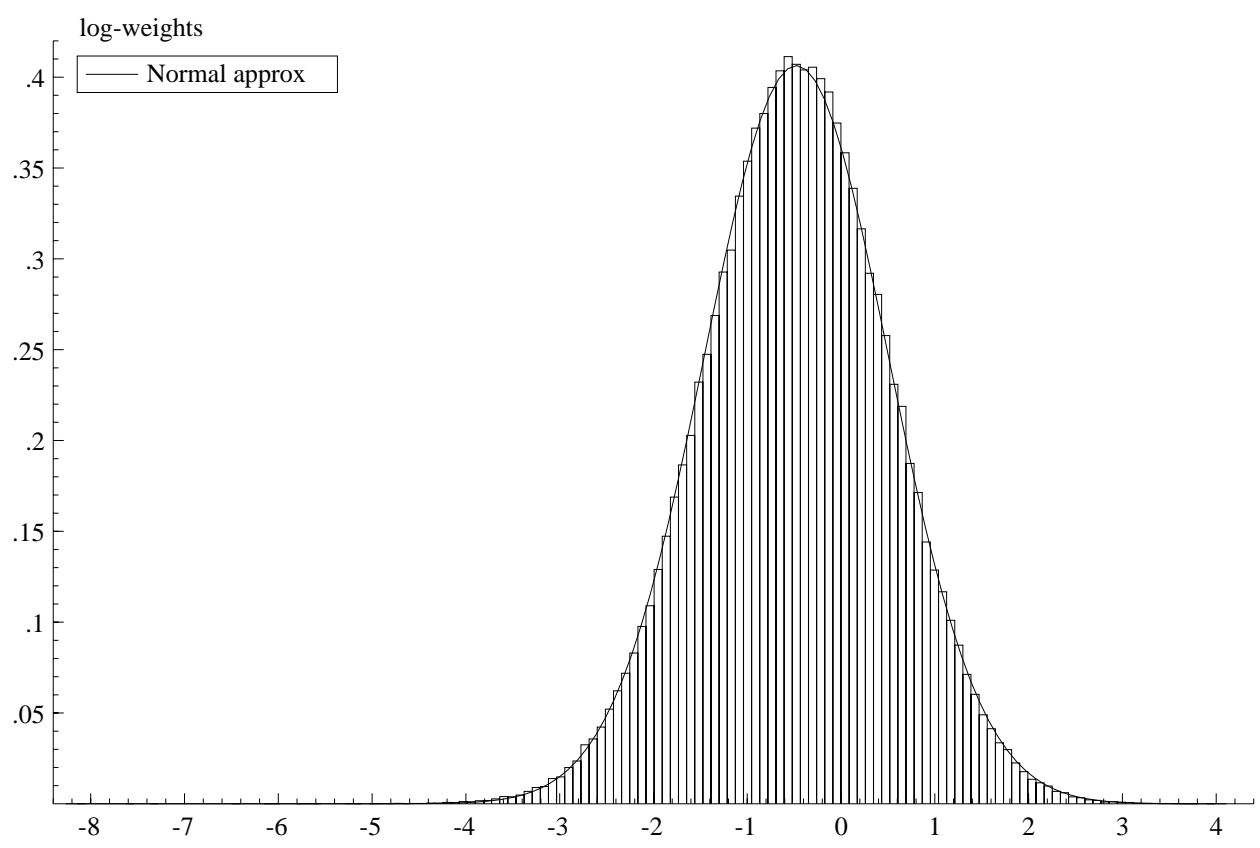

Figure 6: Histogram of the log of the $M \times c^{j}$ for 250,000 sweeps for the integration sampler and a corresponding approximating normal density with fitted mean and standard deviation. All the weights around zero would indicate a perfect sampler.

To see the effect of the weights on the parameters estimates, we reweighted the 250,000 samples displayed in Figure 5. This produced the estimates which are given in Table 7. These Monte Carlo estimates of the posterior means are statistically insignificantly different from Monte Carlo estimated values given in Table 1. However, the Monte Carlo precision has improved dramatically. Further, the Monte Carlo standard errors indicate that this data set could be routinely analysed using around 1,500 sweeps. 


\begin{tabular}{lllrrrr}
\hline & Mean & MC S.E. & Inefficiency & \multicolumn{3}{c}{ Covariance \& Correlation } \\
$\phi \mid y$ & 0.97752 & $7.0324 \mathrm{e}-005$ & 11.20 & 0.00010973 & -0.685 & 0.204 \\
$\sigma_{\eta} \mid y$ & 0.15815 & 0.00024573 & 14.81 & -0.00022232 & 0.00096037 & -0.129 \\
$\beta \mid y$ & 0.64909 & 0.00025713 & 1.64 & 0.00021181 & -0.00039768 & 0.0098312 \\
\hline Time & 10105 & 4.0423 & & & & \\
\hline
\end{tabular}

Table 7: Daily returns for Sterling against Dollar. Summaries of reweighted sample of 250,000 sweeps of the integration sampler. The Monte Carlo S.E. of simulation is computed using a block one tenth of the size of the simulation. Italics are correlations rather than covariances of the posterior. Computer time is seconds on a Pentium Pro/200. The other time is the number of seconds to perform 100 complete passes of the sampler.

This conclusion seems to hold up for some other exchange rate series. Table 8 reports the estimates of the parameters and simulation inefficiency measures for the DM, Yen and Swiss Franc series. This table is the exact analog of Table 2 for the single move algorithm.

\begin{tabular}{lllllll}
\hline & \multicolumn{3}{c}{$\phi \mid y$} & \multicolumn{2}{c}{$\sigma_{\eta} \mid y$} & \multicolumn{2}{c}{$\beta \mid y$} \\
Series & Mean & Inefficiency & Mean & Inefficiency & Mean & Inefficiency \\
DM & 0.96529 & 8.31 & 0.15812 & 11.99 & 0.65071 & 9.73 \\
Yen & 0.97998 & 23.10 & 0.12503 & 35.66 & 0.53534 & 2.71 \\
SwizF & 0.95276 & 13.52 & 0.20738 & 15.33 & 0.70675 & 8.38 \\
\hline
\end{tabular}

Table 8: Bandwidth for each parameter was 100 on all series. In all cases 250,000 sweeps were used.

\section{FILTERING, DIAGNOSTICS AND LIKELIHOOD EVALU- ATION}

\subsection{Introduction}

There has been considerable recent work on the development of simulation based methods to perform filtering, that is computing features of $h_{t} \mid Y_{t}, \theta$, for each value of $Y_{t}=\left(y_{1}, \ldots, y_{t}\right)$. Leading papers in this field include Gordon, Salmond, and Smith (1993), Kitagawa (1996), Isard and Blake (1996), Berzuini, Best, Gilks, and Larizza (1997), West (1993) and Muller (1991). We work with a simple approach which is a special case of a suggestion made by Pitt and Shephard (1997). Throughout we will assume $\theta$ is known. In practice $\theta$ will be set to some estimated value, such as the maximum likelihood estimator or the Monte Carlo estimator of the posterior mean.

The objective is to obtain a sample of draws from $h_{t} \mid Y_{t}, \theta$ given a sample of draws $h_{t-1}^{1}, \ldots, h_{t-1}^{M}$ from $h_{t-1} \mid Y_{t-1}, \theta$. Such an algorithm is called a particle filter in the literature. We now show 
how this may be done. From Bayes theorem,

$$
f\left(h_{t} \mid Y_{t}, \theta\right) \propto f\left(y_{t} \mid h_{t}, \theta\right) f\left(h_{t} \mid Y_{t-1}, \theta\right)
$$

where

$$
f\left(h_{t} \mid Y_{t-1}, \theta\right)=\int f\left(h_{t} \mid h_{t-1}, \theta\right) f\left(h_{t-1} \mid Y_{t-1}, \theta\right) d h_{t-1}
$$

and $f\left(h_{t} \mid h_{t-1}, \theta\right)=f_{N}\left(h_{t} \mid \mu+\phi\left(h_{t-1}-\mu\right), \sigma_{\eta}^{2}\right)$ is the normal evolution density. The latter integral can be estimated from the sample $h_{t-1}^{1}, \ldots, h_{t-1}^{M}$ leading to the approximations

$$
f\left(h_{t} \mid Y_{t-1}, \theta\right) \simeq \frac{1}{M} \sum_{j=1}^{M} f\left(h_{t} \mid h_{t-1}^{j}, \theta\right)
$$

and

$$
f\left(h_{t} \mid Y_{t}, \theta\right) \dot{\propto} f\left(y_{t} \mid h_{t}, \theta\right) \frac{1}{M} \sum_{j=1}^{M} f\left(h_{t} \mid h_{t-1}^{j}, \theta\right) .
$$

The question now is to sample $h_{t}$ from the latter density. The obvious importance sampling procedure of producing a sample $\left\{h_{t}^{j}\right\}$ from $f\left(h_{t} \mid h_{t-1}^{j}, \theta\right)$ and then resampling these draws with weights proportional to $\left\{f\left(y_{t} \mid h_{t}^{j}, \theta\right)\right\}$ is not efficient. An improved procedure runs as follows. Let $h_{t \mid t-1}=\mu+\phi\left(M^{-1} \sum h_{t-1}^{j}-\mu\right)$ and $\log f\left(y_{t} \mid h_{t}, \theta\right)=$ const $+\log f^{*}\left(y_{t}, h_{t}, \theta\right)$. Now expand $\log f^{*}\left(y_{t}, h_{t}, \theta\right)$ in a Taylor series around the point $h_{t \mid t-1}$ as

$$
\begin{aligned}
\log f^{*}\left(y_{t}, h_{t}, \theta\right) & =-\frac{1}{2} h_{t}-\frac{y_{t}^{2}}{2}\left\{\exp \left(-h_{t}\right)\right\} \\
& \leq-\frac{1}{2} h_{t}-\frac{y_{t}^{2}}{2}\left\{\exp \left(-h_{t \mid t-1}\right)\left(1+h_{t \mid t-1}\right)-h_{t} \exp \left(-h_{t \mid t-1}\right)\right\} \\
& =\log g^{*}\left(h_{t}, h_{t \mid t-1}, \theta\right) .
\end{aligned}
$$

Also, after some algebra it can be shown that

$$
g^{*}\left(h_{t}, h_{t \mid t-1}, \theta\right) f\left(h_{t} \mid h_{t-1}^{j}, \theta\right) \propto \pi_{j} f_{N}\left(h_{t} \mid h_{t \mid t-1}^{j}, \sigma_{\eta}^{2}\right)
$$

where

$$
\begin{aligned}
\pi_{j} & =\exp \left\langle\frac{1}{2 \sigma_{\eta}^{2}}\left[\left\{\mu+\phi\left(h_{t-1}^{j}-\mu\right)\right\}^{2}-h_{t \mid t-1}^{j 2}\right]\right\rangle \text { and } \\
h_{t \mid t-1}^{j} & =\mu+\phi\left(h_{t-1}^{j}-\mu\right)+\frac{\sigma_{\eta}^{2}}{2}\left\{y_{t}^{2} \exp \left(-h_{t \mid t-1}\right)-1\right\} .
\end{aligned}
$$

Hence, the kernel of the target density in (14) can be bounded as

$$
f^{*}\left(y_{t}, h_{t}, \theta\right) \frac{1}{M} \sum_{j=1}^{M} f\left(h_{t} \mid h_{t-1}^{j}, \theta\right) \leq g^{*}\left(h_{t}, h_{t \mid t-1}, \theta\right) \frac{1}{M} \sum_{j=1}^{M} f\left(h_{t} \mid h_{t-1}^{j}, \theta\right),
$$

where the right hand side terms are proportional to $\frac{1}{M} \sum_{j=1}^{M} \pi_{j} f_{N}\left(h_{t} \mid h_{t \mid t-1}^{j}, \sigma_{\eta}^{2}\right)$ due to (15). 
These results suggest a simple accept-reject procedure for drawing $h_{t}$. First, we draw a proposal value $h_{t}$ from the mixture density $\frac{1}{M} \sum_{j=1}^{M} \pi_{j}^{*} f_{N}\left(h_{t} \mid h_{t \mid t-1}^{j}, \sigma_{\eta}^{2}\right)$, where $\pi_{j}^{*}=\pi_{j} / \sum_{j} \pi_{j}$. Second, we accept this value with probability $f^{*}\left(y_{t}, h_{t}, \theta\right) / g^{*}\left(h_{t}, h_{t \mid t-1}, \theta\right)$. If the value is rejected, we return to the first step and draw a new proposal.

By selecting a large $M$ this filtering sampler will become arbitrarily accurate.

\subsubsection{Application}

To illustrate this, we apply these methods to the Sterling/Dollar series, filtering the volatility. Throughout we will employ $M=2,500$. Similar results were obtained when $M$ fell to 1,000 , although reducing $M$ below that figure created important biases. The results are made conditional of the estimated parameters, which are taken from Table 9 and based on 2,500 sweeps of the integration sampler.

\begin{tabular}{lllrrrr}
\hline & Mean & MC S.E. & Inefficiency & \multicolumn{3}{c}{ Covariance \& Correlation } \\
$\phi \mid y$ & 0.97611 & 0.0018015 & 11.636 & 0.00014783 & -0.765 & 0.277 \\
$\sigma_{\eta} \mid y$ & 0.16571 & 0.0065029 & 17.657 & -0.00033148 & 0.0012693 & -0.232 \\
$\beta \mid y$ & 0.64979 & 0.0047495 & 1.4563 & 0.00030503 & -0.00074971 & 0.008209 \\
\hline Time & 97.230 & 3.8892 & & & & \\
\hline
\end{tabular}

Table 9: Daily returns for Sterling series. Summaries of reweighted sample of 2,500 sweeps of the integration sampler. The Monte Carlo S.E. of simulation is computed using a block one tenth of the size of the simulation. Italics are correlations rather than covariances of the posterior. Computer time is seconds on a Pentium Pro/200. The other time is the number of seconds to perform 100 complete passes of the sampler.

The resulting filtered and smoothed estimates of the volatility are given in Figure 7, together with a graph of the absolute values of the returns. The graph shows the expected feature of the filtered volatility lagging the smoothed volatility. Throughout the sample, the filtered volatility is slightly higher than the smoothed values due to the gradual fall in volatility observed for these series during this period.

\subsection{Diagnostics}

Having designed a filtering algorithm it is a simple matter to sample from the one-step-ahead prediction density and distribution function. By definition the prediction density is

$$
f\left(y_{t+1} \mid Y_{t}, \theta\right)=\int f\left(y_{t+1} \mid Y_{t}, h_{t+1}, \theta\right) f\left(h_{t+1} \mid Y_{t}, h_{t}, \theta\right) f\left(h_{t} \mid Y_{t}, \theta\right) d h_{t+1} d h_{t}
$$



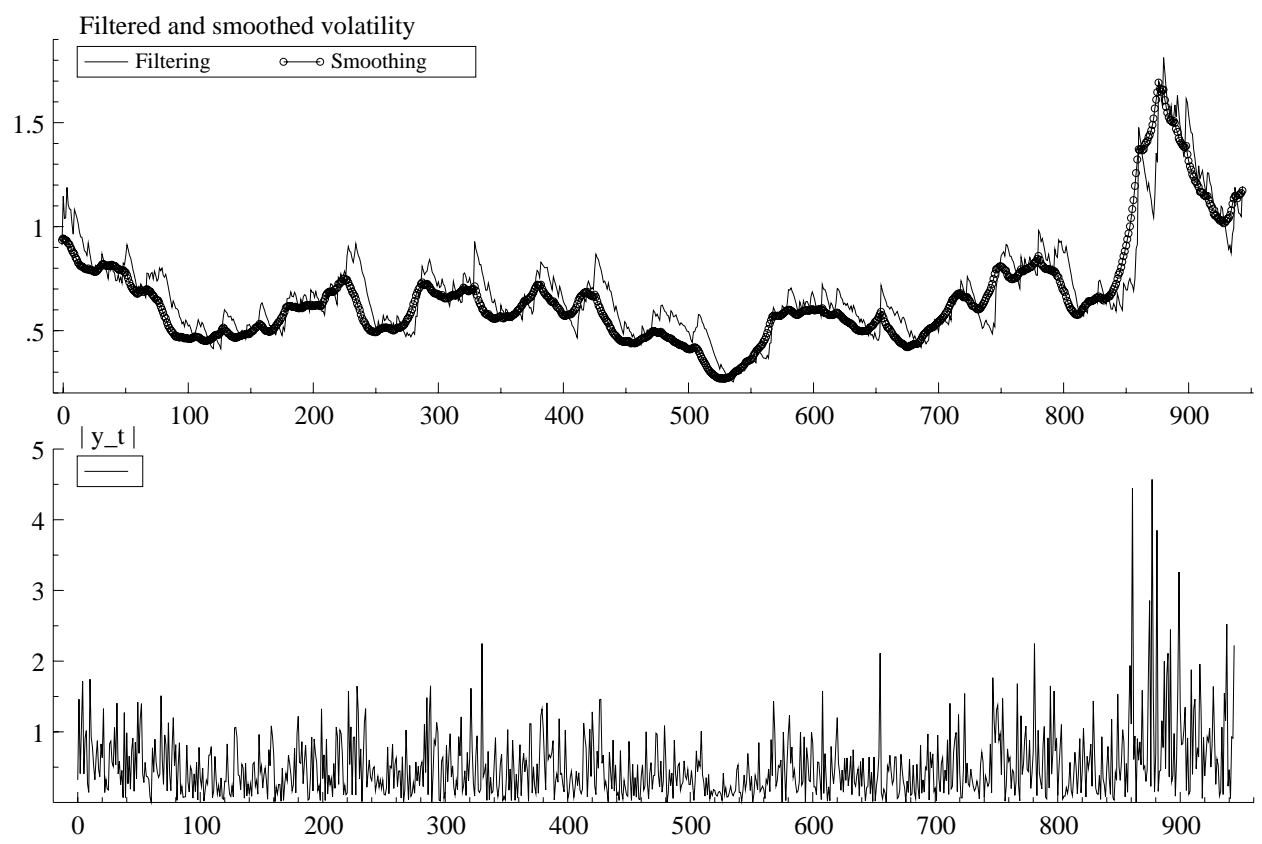

Figure 7: Top: filtered and smoothed estimate of the volatility $\exp \left(h_{t} / 2\right)$, computed using $M=$ 2000. Bottom: $\left|y_{t}\right|$, the absolute values of the returns.

which can be sampled by the method of composition as follows. For each value $h_{t}^{j}(j=1,2, \ldots, M)$ from the filtering algorithm, one samples $h_{t+1}^{j}$ from

$$
h_{t+1}^{j} \mid h_{t}^{j} \sim \mathcal{N}\left\{\mu+\phi\left(h_{t}^{j}-\mu\right), \sigma_{\eta}^{2}\right\}
$$

Based on these $M$ draws on $h_{t+1}$ from the prediction density, we can estimate the probability that $y_{t+1}^{2}$ will be less than the observed $y_{t+1}^{o 2}$ :

$$
\operatorname{Pr}\left(y_{t+1}^{2} \widehat{\leq y_{t+1}^{o 2}} \mid Y_{t}, \theta\right)=u_{t+1}^{M}=\frac{1}{M} \sum_{j=1}^{M} \operatorname{Pr}\left(y_{t+1}^{2} \leq y_{t+1}^{o 2} \mid h_{t+1}^{j}, \theta\right)
$$

For each $t=1, \ldots, n$, under the null of a correctly specified model $u_{t}^{M}$ converges in distribution to independent and identically distributed uniform random variables as $M \rightarrow \infty$ (Rosenblatt (1952)). This provides a valid basis for diagnostic checking. These variables can be mapped into the normal distribution, by using the inverse of the normal distribution function $n_{t}^{M}=F^{-1}\left(u_{t}^{M}\right)$ to give a standard sequence of independent and identically distributed normal variables, which are then transformed one-step-ahead forecasts normed by their correct standard errors. These can be used to carry out Box-Ljung, normality, and heteroscedasticity tests, among others.

The computed forecast uniforms and resulting correlograms and QQ plots are given in Figure 8. The results suggest the model performs quite well, although it reveals some outliers. However, 

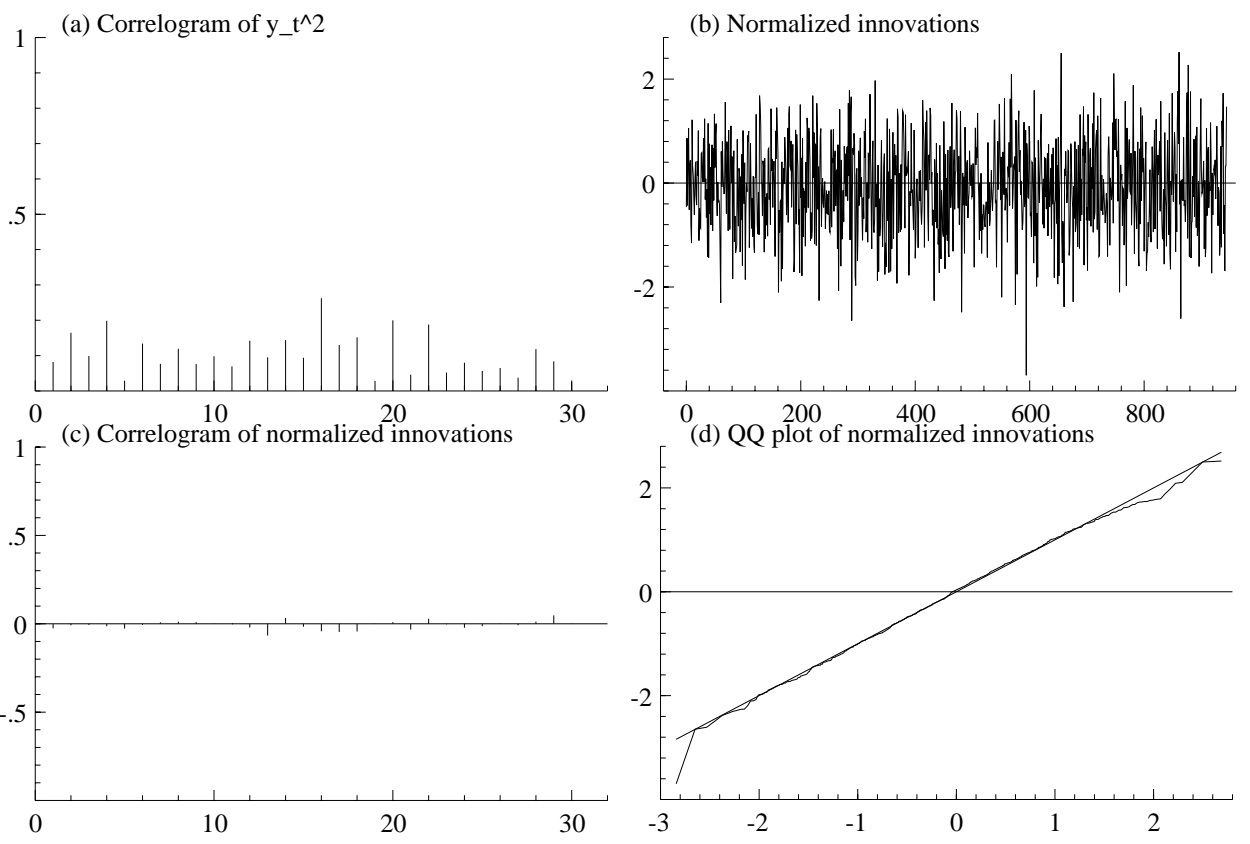

Figure 8: Diagnostic checks. Graph (a): correlogram of $y_{t}^{2}$. Graph (b): normalised innovations. Graph (c): the corresponding correlogram. Graph (d): associated QQ-plot.

closer inspection shows that the outliers correspond to small values of $y_{t}^{2}$. This suggests that the SV model fails to accommodate some of the data values that have limited daily movements. On the other hand it appears to perform well when the movements in the data are large. This will be made more formal in the next sub-section.

\subsubsection{Likelihood estimation}

The one-step-ahead predictions can also be used to estimate the likelihood function since the one-step-ahead prediction density, $f\left(y_{t+1} \mid Y_{t}\right)$, can be estimated as:

$$
\frac{1}{M} \sum_{j=1}^{M} f\left(y_{t+1} \mid h_{t+1}^{j}\right), \quad h_{t+1}^{j} \mid h_{t}^{j} \sim \mathcal{N}\left\{\mu+\phi\left(h_{t}^{j}-\mu\right), \sigma_{\eta}^{2}\right\},
$$

using drawings from the filtering simulator. The same argument gives a filtered estimate of $h_{t+1}$ using the information up to time $t$.

Table 10 shows the results from some standard diagnostic checks on the $n_{1}^{M}, \ldots, n_{n}^{M}$ produced by the fitted model. Under the correctness of the model, the diagnostics should indicate that the variables are Gaussian white noise. We report the skewness and kurtosis coefficients,

$$
\text { Skew }=\frac{n b_{3}}{6}, \quad \text { Kurtosis }=\frac{n\left(b_{4}-3\right)^{2}}{24},
$$


where $b_{3}$ and $b_{4}$ denote the standardized estimators of the third and fourth moment of $\left\{n_{t}^{M}\right\}$ about the mean, an overall Bowman and Shenton (1975) normality statistic which combines these two measures and the Box-Ljung statistic using 30 lags. The Table also gives the simulation standard error for these statistics, based on repeating the simulation ten times with different random draws but with the data fixed. Finally, for comparison the Table gives the same diagnostics for the $\mathcal{N}\left(0, \sigma^{2}\right)$ and scaled Student t iid models. The results suggest that there are no straightforward failures in the way the model has been fitted.

\begin{tabular}{cccccc}
\hline & Skew & Kurtosis & Normality & BL(30) & log-like \\
SV & 1.4509 & 0.54221 & 2.3992 & 18.555 & -918.56 \\
& $(0.057)$ & $(0.083)$ & $(0.295)$ & $(0.120)$ & $(0.558)$ \\
\hline NID & 11.505 & 21.640 & 600.65 & 401.20 & -1018.2 \\
tID(4.87) & 1.2537 & 1.2156 & 3.0494 & 700.62 & -964.56
\end{tabular}

Table 10: Diagnostics of the $S V$ model using $M=2,500 . B L(l)$ denotes a Box-Ljung statistic on $l$ lags. The figures in brackets are simulation standard errors using 10 replications. The two other models are fitted using $M L$. The estimated degrees of the Student $t$ model is given in brackets.

\section{COMPARISON OF NON-NESTED MODELS VIA SIMU- LATION}

\subsection{GARCH model}

In this section we compare the fit of basic SV models with the GARCH models commonly used in the literature. Two approaches are used in this non-nested model comparison - one based on likelihood ratios and another based on ratios of marginal likelihoods resulting in what are called Bayes factors.

The notation we use for the Gaussian $\operatorname{GARCH}(1,1)$ model is:

$$
y_{t} \mid Y_{t-1} \sim \mathcal{N}\left(0, \sigma_{t}^{2}\right), \text { where } \sigma_{t}^{2}=\alpha_{0}+\alpha_{1} y_{t-1}^{2}+\alpha_{2} \sigma_{t-1}^{2}
$$

while the equivalent Student - t model introduced by Bollerslev (1987) is denoted as t-GARCH with $\nu$ as the notation for the positive degrees of freedom.

The diagnostic statistics given in Table 11 suggest that the Gaussian GARCH model does not fit the data very well, suffering from positive skewness and excess kurtosis. This suggests that the model cannot accommodate the extreme positive observations in the data. The t-GARCH model is better, with much better distributional behaviour. Again its diagnostics for serial dependence are satisfactory. The fitted likelihood is very slightly better than the SV model, although it has one more parameter. 


\begin{tabular}{cccccccc}
\hline & $\alpha_{0}$ & $\alpha_{1}+\alpha_{2}$ & Skew & kurt & Normality & BL(30) & log-like \\
GARCH & 0.0086817 & 0.98878 & 4.5399 & 4.3553 & 39.580 & 16.183 & -928.13 \\
t-GARCH (8.44) & 0.0058463 & 0.99359 & 0.56281 & 0.31972 & 0.41897 & 22.515 & -917.22 \\
\hline
\end{tabular}

Table 11: Diagnostics of the ML estimators of the Gaussian and Student $t$ distributed GARCH models. BL(l) denotes a Box-Ljung statistic on l lags. Above the line are the answers of the real data, the ones below are the corrected observations. Figures in brackets for the $t$-GARCH model are the estimated degrees of freedom.

\subsection{Likelihood ratio statistics}

There is an extensive literature on the statistical comparison of non-nested models based on likelihood ratio statistics. Much of the econometric literature on this topic is reviewed in Gourieroux and Monfort (1994). The approach we suggest here relies on simulation and is based on Atkinson (1986). Related ideas appear in, for instance, Pesaran and Pesaran (1993) and Hinde (1992).

Let $\mathcal{M}_{1}$ denote the SV model and $\mathcal{M}_{0}$ the GARCH model. Then, the likelihood ratio test statistic for comparative fit that is investigated here is given by

$$
L R_{y}=2\left\{\log \widehat{f}\left(y \mid \mathcal{M}_{1}, \hat{\theta}_{1}\right)-\log f\left(y \mid \mathcal{M}_{0}, \tilde{\theta}_{0}\right)\right\}
$$

where $\log \widehat{f}\left(y \mid \mathcal{M}_{1}, \hat{\theta}_{1}\right)$ and $\log f\left(y \mid \mathcal{M}_{0}, \widetilde{\theta}_{0}\right)$ denote the respective estimates of the log likelihoods, the former estimated by simulation as described above ${ }^{8}, \widehat{\theta}_{1}$ is the estimated posterior mean of SV model parameters and $\widetilde{\theta}_{0}$ the MLE of the GARCH model parameters. The sampling variation of $L R_{y}$ under the hypothesis that the SV model is true or under the alternative that the GARCH model is true is approximated by simulation, following Atkinson (1986). Clearly, analytical derivations of the sampling distribution are difficult given the unconventional estimators of the log-likelihood.

Under the assumption that the SV model is true and the true values of its parameters are $\theta_{1}^{(0)}$, we generate simulations $y^{i}, i=1, \ldots, M$ from the true model. For each simulated series we estimate the parameters of the GARCH and SV models and record the value of $L R_{y}$, which we denote as $L R_{y}^{i}$. The resulting scatter of values $L R_{y}^{1}, \ldots, L R_{y}^{M}$ are a sample from the exact distribution of $L R_{y}$ under the SV null. The fact that we estimated the likelihood and the parameters of the SV model for each $y^{i}$ does not alter this result. Hence we could use these simulations $L R_{y}^{i}$ as inputs into a trivial Monte Carlo test (see, for example, Ripley (1987, p. 171-4)) of the hypothesis that the GARCH model is true. Unfortunately $\theta_{1}^{(0)}$ is unknown and so

\footnotetext{
${ }^{8}$ The GARCH process has to be initialized by setting $\sigma_{0}^{2}$. The choice of this term effects the likelihood function. In our calculations we set $\sigma_{0}^{2}=\alpha_{0} /\left(1-\alpha_{1}-\alpha_{2}\right)$.
} 
it is estimated from the data and chosen to be $\widehat{\theta}_{1}$. This introduces an additional approximation error into the sampling calculation which falls as the sample size $n \rightarrow \infty$.

The estimated approximate sampling distributions of $L R_{y}$ under each hypothesis based on 99 simulations plus the realization from the data are given in Figure 9. This figure shows that if the null of the SV model is true, then $L R_{y}$ can be expected to be positive when the alternative is a Gaussian GARCH, while it is expected to be around zero when the alternative is a t-GARCH.

For the Sterling series the observed $L R_{y}$ is 19.14 for the SV model against GARCH and -2.68 for the SV model against t-GARCH. This suggests that the SV model fits the data better than the GARCH model but slightly worse than the t-GARCH model (which has one more parameter). These results are confirmed by looking at the simulated $L R_{y}$. Table 12 records the ranking of the observed $L R_{y}$ amongst the 99 simulations conducted under the assumption that the SV model is true. Hence if the observed $L R_{y}$ is the 96th largest, then it is ranked as being 96th. If the ranking is either close to zero or 100 then this would provide evidence against the SV model.

The recorded rankings under the SV hypothesis are not very extreme, with about $20 \%$ of the simulations generating LR tests against the GARCH model which are higher than that observed, while $30 \%$ of the simulations were lower than that observed for the t-GARCH LR test. Although suggestive, neither of these tests are formally significant. This implies that they are both consistent with the SV model being true.

A more decisive picture is generated when the Gaussian GARCH model is the null hypothesis. No value is as extreme as the observed LR test against the SV model, rejecting the Gaussian GARCH model for these data. The evidence of the test against the t-GARCH model is less strong.

In summary, the observed non-nested $L R_{y}$ tests give strong evidence against the use of Gaussian GARCH models. The two remaining models are the t-GARCH and SV models. The statistics show a slight preference for the t-GARCH model, but this model is less parsimonious than the SV model and so it would be fairer to argue for the statement that they fit the data more or less equally well. These results carry over to the other three exchange rates. The results from the non-nested tests are given in Table 12, although there is a considerable evidence that the t-GARCH model is preferable to the SV model for the Yen series.

\subsection{Bayes factors}

An alternative to likelihood ratio statistics is the use of Bayes factors, which are symmetric in the models and extremely easy to interpret. The approach adopted here for the computation of Bayes factors relies on the method developed by Chib (1995). From the basic marginal likelihood 
(a) null: SV, alternative: Gaussian GARCH

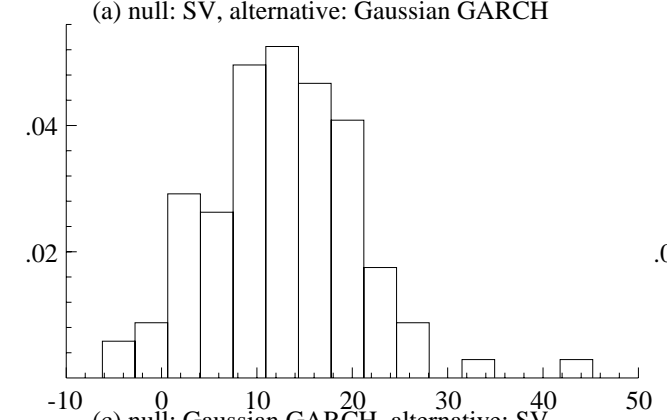

(b) null: SV, alternative: t-GARCH
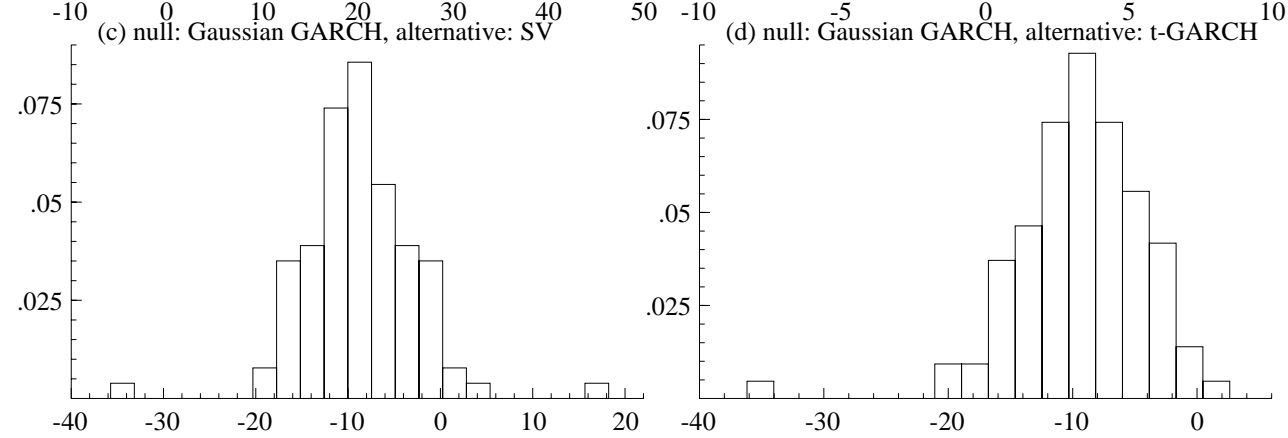

Figure 9: Non-nested testing. Graphs (a)-(b) LR computed when $S V$ is true. Graph (a): SV against a GARCH model. Graph (b): SV against a t-GARCH. The observed values are 19.14 and -2.68 respectively, which are 80th and 29th out of the 100 samples. Graphs (c)-(d): LR computed when GARCH model is true. Graph (c): GARCH against SV. Graph (d): GARCH against $t$-GARCH. The observed values are 19.14 and -2.68 respectively, which ranks them 100 th and 79th out of the 100 samples.

identity in Chib (1995), the log of the Bayes factor can be written as

$$
\begin{aligned}
& \log f\left(y \mid \mathcal{M}_{1}\right)-\log f\left(y \mid \mathcal{M}_{0}\right) \\
= & \left\{\log f\left(y \mid \mathcal{M}_{1}, \theta_{1}^{*}\right)+\log f\left(\theta_{1}^{*} \mid \mathcal{M}_{1}\right)-\log f\left(\theta_{1}^{*} \mid \mathcal{M}_{1}, y\right),\right\} \\
& -\left\{\log f\left(y \mid \mathcal{M}_{0}, \theta_{0}^{*}\right)+\log f\left(\theta_{0}^{*}\right)-\log f\left(\theta_{0}^{*} \mid \mathcal{M}_{0}, y\right)\right\}
\end{aligned}
$$

for any values of $\theta_{0}^{*}$ and $\theta_{1}^{*}$. Here $f\left(\theta_{0}^{*}\right)$ is the GARCH prior density, while $f\left(\theta_{1}^{*} \mid \mathcal{M}_{1}\right)$ is the prior for the SV parameters. The likelihood for the GARCH model is known, while that of the SV model is estimated via simulation as described above. Next, the posterior densities $f\left(\theta_{0}^{*} \mid \mathcal{M}_{0}, y\right)$ and $f\left(\theta_{1}^{*} \mid \mathcal{M}_{1}, y\right)$ are estimated at the single points $\theta_{0}^{*}$ and $\theta_{1}^{*}$ using a Gaussian kernel applied to the posterior sample of the parameters. We follow the suggestion in Chib (1995) and use the posterior means of the parameters as $\theta_{0}^{*}$ and $\theta_{1}^{*}$ since the choice of these points is arbitrary.

To perform a Bayes estimation of the GARCH model we have to write down some priors for the GARCH parameters. This is most easily done by representing the model in its ARMA $(1,1)$ form for squared data:

$$
y_{t}^{2}=\alpha_{0}+\left(\alpha_{1}+\alpha_{2}\right) y_{t-1}^{2}+v_{t}-\alpha_{2} v_{t-1}, \quad v_{t}=\left(\varepsilon_{t}^{2}-1\right) \sigma_{t}^{2} .
$$




\begin{tabular}{cccc|ccc} 
series & \multicolumn{3}{c}{ SV verses GARCH } & \multicolumn{3}{c}{ SV against t-GARCH } \\
& observed & rank SV & rank GARCH & observed & rank SV & rank GARCH \\
Sterling & 19.14 & $81 \mathrm{st}$ & 100 th & -2.68 & 29 th & 79 th \\
DM & 11.00 & $61 \mathrm{st}$ & 100 th & -3.84 & 9 th & 87 th \\
Yen & 19.84 & 99 th & 100 th & -30.50 & 1 st & 1 st \\
SwizF & 53.12 & 100 th & 100th & -3.62 & 20 th & 98 th \\
\hline
\end{tabular}

Table 12: Non-nested LR tests of the SV model against the ARCH models. In each case the 99 simulations were added to the observed $L R_{y}$ to form the histograms. The reported $r$-th rankings are the r-th largest of the observed LR test out of the $100 L_{2}$ tests conducted under $S V$ or GARCH model.

Hence $\alpha_{1}+\alpha_{2}$ is the persistence parameter, $\alpha_{2}$ (which has to be positive) is the negative of the moving average coefficient, while $\alpha_{0} /\left(1-\alpha_{1}-\alpha_{2}\right)$ is the unconditional expected value of $y_{t}^{2}$. We will place the same prior on $\alpha_{1}+\alpha_{2}$ as was placed on the persistence parameter $\phi$ in the SV model (see (5)). This will force the GARCH process to be covariance stationary. The prior specification is completed by assuming that $\alpha_{2} /\left(\alpha_{1}+\alpha_{2}\right) \mid \alpha_{1}+\alpha_{2}=r_{\alpha}$ follows a Beta distribution with

$\log f\left\{\alpha_{2} /\left(\alpha_{1}+\alpha_{2}\right) \mid \alpha_{1}+\alpha_{2}=r_{\alpha}\right\}=$ const $+\left\{\phi^{(1)}-1\right\} \log \left\{\frac{\alpha_{2}}{r_{\alpha}}\right\}+\left\{\phi^{(2)}-1\right\} \log \left\{\frac{r_{\alpha}-\alpha_{2}}{r_{\alpha}}\right\}$.

Since we would expect that $\alpha_{2} /\left(\alpha_{1}+\alpha_{2}\right)$ to be closer to one than zero, we will take $\phi^{(1)}=45$ and let $\phi^{(2)}=2$. This gives a mean of 0.957 . The scale parameter $\alpha_{0} /\left(1-\alpha_{1}-\alpha_{2}\right) \mid \alpha_{1}, \alpha_{2}$ are given a standard diffuse inverse chi-squared prior distribution. Finally, for the t-GARCH model, $v-2$ was given in chi-squared prior with a mean of ten.

GARCH

\begin{tabular}{cccc|cccc} 
series & $\alpha_{1}+\alpha_{2}$ & $\log f_{\text {GARCH }}$ & $\log$ Bayes & $\alpha_{1}+\alpha_{2}$ & $\mathrm{v}$ & $\log f_{\text {GARCH }}$ & $\log$ Bayes \\
Sterling & 0.9802 & -928.64 & 9.14 & 0.9822 & 9.71 & -918.13 & -3.512 \\
DM & 0.9634 & -952.88 & 6.52 & 0.9712 & 12.82 & -945.63 & -2.688 \\
Yen & 0.9850 & -798.79 & 13.22 & 0.9939 & 6.86 & -774.8 & -11.28 \\
SwizF & 0.9153 & -1067.2 & 27.86 & 0.9538 & 7.57 & -1039.0 & -0.84 \\
\hline
\end{tabular}

Table 13: Estimated Bayes factors for SV model against GARCH model and t-GARCH. All the densities were evaluate at the estimated posterior mean.

In order to carry out the MCMC sampling we used the Gilks, Best, and Tan (1995) procedure which just requires the programming of the priors and the GARCH likelihood.

The results of the calculations are given in Table 13 . They are very much in line with the likelihood ratio analysis given in Table 12. Again the SV model dominates the Gaussian GARCH model, while it suffers in comparison with the t-GARCH model, especially for the 
Yen data series. It should be mentioned, however, that these conclusions are in relation to the simplest possible SV model. The performance of the SV model can be improved by considering other versions of the model, for example, one that relaxes the Gaussian assumption. We discuss this and other extensions next.

\section{EXTENSIONS}

\subsection{More complicated dynamics}

This paper has suggested three ways of performing Bayesian analysis of the SV model: single move, offset mixture and integration sampling. All three extend to the problem where the volatility follows a more complicated stochastic process than an $A R(1)$. A useful framework is:

$$
h_{t}=c_{t}+Z_{t} \gamma_{t}, \quad \text { where } \quad \gamma_{t+1}=d_{t}+T_{t} \gamma_{t}+H_{t} u_{t}
$$

where $u_{t} \stackrel{i i d}{\sim} \mathcal{N}(0, I), c_{t}$ and $d_{t}$ are assumed to be strictly exogenous, and $Z_{t}, T_{t}$ and $H_{t}$ are selected to represent the log-volatility appropriately. With this framework the log volatility process can be specified to follow an ARMA process.

In the single move Gibbs algorithm, it is tempting to work with the $\gamma_{t}$ as

$$
f\left(\gamma_{t} \mid y_{t}, \gamma_{t-1}, \gamma_{t+1}\right) \propto f\left(y_{t} \mid c_{t}+Z_{t} \gamma_{t}\right) f\left(\gamma_{t} \mid \gamma_{t-1}\right) f\left(\gamma_{t+1} \mid \gamma_{t}\right)
$$

has a simple structure. However, this would suffer from the problems of large MCMC simulation inefficiency documented above especially if $\gamma_{t}$ is high dimensional or if the $\left\{\gamma_{t}\right\}$ process displayed considerable memory (akin to the example given in Carter and Kohn (1994)). Alternatively, one could sample $h_{t}$ using

$$
f\left(h_{t} \mid h_{\backslash t}, y\right) \propto f\left(y_{t} \mid h_{t}\right) f\left(h_{t} \mid h_{\backslash t}\right),
$$

as we can evaluate $h_{t} \mid h_{\backslash t}$ using the de Jong (1998) scan sampler. This is uniformly superior to the algorithms built using (20). Neither of these choices would be competitive, however, with versions of the multi-move and integration sampler which rely on the state space form and can thus be trivially extended to cover these models.

More sophisticated dynamics for the volatility could be modeled by exploiting factor type models. An example of this is

$$
h_{t}=h_{1 t}+h_{2 t}, \quad h_{1 t+1}=\phi_{1} h_{1 t}+\eta_{1 t}, \quad h_{2 t+1}=\phi_{2} h_{2 t}+\eta_{2 t},
$$

where $\phi_{1}>\phi_{2}$ and $\eta_{1 t}, \eta_{2 t}$ are independent Gaussian white noise processes. Here $h_{1 t}$ and $h_{2 t}$ would represent the longer-term and shorter-term fluctuations in log-volatility. The introduction of such components, appropriately parametrized, produce volatility versions of the long memory models advocated by Cox (1991). 


\subsection{Missing observations}

The framework described above can also be extended to handle missing data. Suppose that the exchange rate $r_{34}$ at time 34 is missing. Then the returns $y_{34}$ and $y_{35}$ would be missing. We could complete the data by adding in $r_{34}$ to the list of unknowns in the sampling. Given $r_{34}$ we could generate $y$ and then sweep $h, \theta \mid y$. Having carried this out we could update $r_{34}$ by drawing it given $h, \theta$ and $y$. Iterating this procedure gives a valid MCMC algorithm and so would efficiently estimate $\theta$ from the non-missing data.

This argument generalizes to any amount of missing data. Hence this argument also generalizes to the experiment where we think of the SV model (1) holding at a much finer discretization than the observed data. Think of the model holding at intervals of $1 / d$-th of a day, while suppose that the exchange rate $r_{t}$ is available daily. Then we can augment the 'missing' intra-daily data $\widetilde{r}_{t}=\left(r_{t_{1}}, \ldots, r_{t_{d-1}}\right)$ to the volatilities $\widetilde{h}_{t}=\left(h_{t_{1}}, \ldots, h_{t_{d-1}}, h_{t}\right)$ and design a simple MCMC algorithm to sample from

$$
\widetilde{r}_{1}, \ldots, \widetilde{r}_{n}, \widetilde{h}_{1}, \ldots, \widetilde{h}_{n}, \theta \mid r_{0}, \ldots, r_{n}
$$

This will again allow efficient estimation of $\theta$ from the 'coarse' daily data even though the model is true at the intra-daily level. This type of argument is reminiscent of the indirect inference methods which have recently been developed for diffusions by Gourieroux, Monfort, and Renault (1993) and Gallant and Tauchen (1996), however our approach has the advantage of not depending on the ad hoc choice of an auxiliary model and is automatically fully efficient.

\subsection{Heavy-tailed SV models}

The discrete time SV model can be extended to allow $\varepsilon_{t}$ in (1) to be more heavy-tailed than the normal distribution. This would help in overcoming the comparative lack of fit indicated by Table 12 for the Yen series. One approach, suggested in Harvey, Ruiz, and Shephard (1994) amongst others, is to use an ad hoc scaled Student t distribution, so that

$$
\varepsilon_{t}=\frac{1}{\sqrt{v-2}} \zeta_{t} / \sqrt{\chi_{t, v}^{2} / v}, \quad \text { where } \quad \zeta_{t} \stackrel{i i d}{\sim} \mathcal{N}(0,1), \quad \chi_{t, v}^{2} \stackrel{i i d}{\sim} \chi_{v}^{2}
$$

and the $\zeta_{t}$ and $\chi_{t, v}^{2}$ are independent of one another. The single move and offset mixture algorithms immediately carry over to this problem if we design a Gibbs sampler for $\chi_{1, v}^{2}, \ldots, \chi_{n, v}^{2}, h, \theta \mid y$ or $\chi_{1, v}^{2}, \ldots, \chi_{n, v}^{2}, h, \theta, \omega \mid y$ respectively.

An alternative to this, which can be carried out in the single move algorithm, would be

to directly integrate out the $\chi_{t, v}^{2}$, which would mean $f\left(y_{t} \mid h_{t}, \theta\right)$ would be a scaled Student $\mathrm{t}$ distribution. This has the advantage of reducing the dimension of the resulting simulation. However, the conditional sampling becomes more difficult. This is because $f\left(y_{t} \mid h_{t}, \theta\right)$ is no 
longer log-concave in $h_{t}$ and the simple accept/reject algorithm will no longer work. However, one could adopt the pseudo-dominating accept/reject procedure that is discussed in Tierney (1994) and Chib and Greenberg (1995). This version of the algorithm incorporates a Metropolis step in the accept/reject method and does not require a bounding function. The same ideas can also be extended for multivariate models and models with correlated $\varepsilon_{t}, \eta_{t}$ errors.

\subsection{Semi-parametric SV}

The offset mixture representation of the SV model naturally leads to a semi-parametric version of the SV model. Suppose we select the "parameters" $m_{1}, \ldots, m_{K}, v_{1}^{2}, \ldots, v_{K}^{2}, q_{1}, \ldots, q_{K}$ freely from the data. Then, this procedure is tantamount to the estimation of the density of the shocks $\varepsilon_{t}$. The constraint that $\operatorname{Var}\left(\varepsilon_{t}\right)=1$ is automatically imposed if $\mu$ is incorporated into these mixture weights.

This generic approach to semi-parametric density estimation along with MCMC type algorithms for the updating of the mixture parameters has been suggested by Escobar and West (1995) and Richardson and Green (1997). Mahieu and Schotman (1997) use a simulated EM approach to estimate a small numbers of mixtures inside an SV model.

\subsection{Prior sensitivity}

The methods developed above can be easily modified to assess the consequences of changing the prior. Instead of rerunning the entire samplers with the alternative prior, one can reweight the simulation output so that it corresponds to the new prior - in much the same way as the simulation was reweighted to overcome the bias caused by the offset mixture. Since the posterior is

$$
f(\theta, h \mid y) \propto f(y \mid h, \theta) f(h \mid \theta) f(\theta)=f(y \mid h, \theta) f(h \mid \theta) f^{*}(\theta) \frac{f(\theta)}{f^{*}(\theta)},
$$

where $f(\theta)$ denotes the new prior and $f^{*}(\theta)$ the prior used in the simulations, the reweighting follows the form of $(12)$ where $w^{j}=\log f\left(\theta^{j}\right)-\log f^{*}\left(\theta^{j}\right)$. This is particularly attractive as the reweighting is a smooth function of the difference between the old prior $f^{*}$ and the new prior $f$. Rerunning the sampler will not have this property.

\subsection{Multivariate factor SV models}

The basis of the $N$ dimensional factor SV model will be

$y_{t}=B f_{t}+\varepsilon_{t}, \quad$ where $\quad\left(\begin{array}{c}\varepsilon_{t} \\ f_{t}\end{array}\right) \sim \mathcal{N}\left\langle 0, \operatorname{diag}\left\{\exp \left(h_{1 t}\right), \ldots, \exp \left(h_{N t}\right), \exp \left(h_{N+1 t}\right), \ldots, \exp \left(h_{N+K t}\right)\right\}\right\rangle$, where $f_{t}$ is $K$ dimensional and

$$
\left(h_{t+1}-\mu\right)=\left(\begin{array}{ll}
\phi_{\varepsilon} & 0 \\
0 & \phi_{f}
\end{array}\right)\left(h_{t}-\mu\right)+\eta_{t}, \quad \eta_{t} \sim \mathcal{N}\left\{0,\left(\begin{array}{ll}
\Sigma_{\varepsilon \eta} & 0 \\
0 & \Sigma_{f \eta}
\end{array}\right)\right\} .
$$


As it stands the model is highly overparameterized. This basic structure was suggested in the factor ARCH models analyzed ${ }^{9}$ by Diebold and Nerlove (1989) and refined by King, Sentana, and Wadhwani (1994), but replaces the unobserved ARCH process for $f_{t}$ by SV processes. It was mentioned as a possible multivariate model by Shephard (1996) and discussed by Jacquier, Polson, and Rossi (1995).

Jacquier, Polson, and Rossi (1995) discussed using MCMC methods on a simplified version ${ }^{10}$ of this model, by exploiting the conditional independence structure of the model to allow the repeated use of univariate MCMC methods to analyse the multivariate model. This method requires the diagonality of $\phi_{\varepsilon}, \phi_{f}, \Sigma_{\varepsilon \eta}$ and $\Sigma_{f \eta}$ to be successful. However, their argument can be generalized in the following way for our offset mixture approach.

Augment the unknown $h, \theta$ with the factors $f$, for then $h \mid f, y, \theta$ has a very simple structure. In our case we can transform each $f_{j t}$ using

$$
\log \left(f_{j t}^{2}+c\right)=h_{N+j t}+z_{j t}, \quad z_{j t} \mid s_{j t}=i \sim \mathcal{N}\left(m_{i}-1.2704, v_{i}^{2}\right)
$$

noting that given the mixtures the $z_{j t}$ are independent over $j$ as well as $t$. Hence we can draw form all at once $h \mid f, s, y, \theta$. This can then be added to routines which draw from $f \mid y, h, \theta$ and $\theta \mid y, h, f$ to complete the sampler.

\section{CONCLUSION}

In this paper we have described a variety of new simulation-based strategies for estimating general specifications of stochastic volatility models. The single move accept/reject algorithm is a natural extension of the previous work in the literature. It is very simple to implement, reliable and is easily generalizable. However, it can have poor convergence properties which has prompted us to develop other samplers which exploit the time series structure of the model.

The key element of our preferred sampler is the linearization made possible by a log-square transformation of the measurement equation and the approximation of a $\log \chi^{2}$ random variable by a mixture of normal variables. This, coupled with the Bayesian re-weighting procedure to correct for the linearization error, enables the analysis of complex models using the wellestablished methods for working with conditionally Gaussian state-space models. The simulation conducted in this paper shows that our proposed methods can achieve significant efficiency gains over previously proposed methods for estimating stochastic volatility models. Furthermore, this approach will continue to perform reliably as we move to models with more complicated dynamics.

\footnotetext{
${ }^{9}$ Using approximate likelihood methods. Exact likelihood methods are very difficult to construct for factor ARCH models.

${ }^{10}$ Their model sets $\phi$ and $\Sigma_{\eta}$ to be diagonal. These constraints are not both needed for identifiability.
} 
The paper also discusses the computation of the likelihood function of SV models which is required in the computation of likelihood ratio statistics and Bayes factors. A formal comparison of the SV model in relation to the popular heavy tailed version of GARCH model is also provided for the first time. An interesting set of methods for filtering the volatilities and obtaining diagnostics for model adequacy are also developed. The question of missing data is also taken up in the analysis. The results in this paper, therefore, provide a unified set of tools for a complete analysis of SV models that includes estimation, likelihood evaluation, filtering, diagnostics for model failure, and computation of statistics for comparing non-nested models. Work continues to refine these results, with the fitting of ever more sophisticated stochastic volatility models.

\section{AVAILABLE SOFTWARE}

All the software used in this paper can be downloaded from the World Wide Web at the URL:

http://www.nuff.ox.ac.uk/users/shephard/ox/

The software is fully documented. We have linked raw $\mathrm{C}++$ code to the graphics and matrix programming language Ox of Doornik (1996) so that these procedures can be easily used by non-experts.

In the case of the single move Gibbs sampler and the diagnostics routines the software is unfortunately specialized to the SV model with $\mathrm{AR}(1)$ log-volatility. However, the other procedures for sampling $h \mid y, s, \theta$ and the resampling weights are general.

\section{ACKNOWLEDGEMENTS}

This paper is an extensively revised version of a paper with the same title by Sangjoon Kim and Neil Shephard. That version of the paper did not have the section on the use of the reweighting which corrects for the mixture approximation, nor the formal non-nested testing procedures for comparison with GARCH models. Neil Shephard would like to thank the ESRC for their financial support through the project 'Estimation via Simulation in Econometrics' and some computational help from Michael K. Pitt and Jurgen A. Doornik. All the authors would like to thank the referees for their comments on the previous version of the paper.

Sangjoon Kim's work was carried out while he was a Ph.D. student at Department of Economics, Princeton University, under the supervision of John Campbell. Sangjoon Kim would like to thank Princeton University for their financial support. The comments of the participants of the 'Stochastic volatility' conference of October 1994, held at HEC (Université de Montreal), are gratefully acknowledged. Finally, Neil Shephard would like to thank A.C.Atkinson and D.R.Cox for various helpful conversations on non-nested likelihood ratio testing. 


\section{APPENDIX}

This appendix contains various algorithms which allow the efficient computations of some of the quantities required in the paper.

\subsection{Basic Gaussian state space results}

We discuss general filtering and simulation smoothing results which are useful for a general Gaussian state space model. We analyse the multivariate model:

$$
\begin{aligned}
y_{t} & =c_{t}+Z_{t} \gamma_{t}+G_{t} u_{t}, \\
\gamma_{t+1} & =d_{t}+T_{t} \gamma_{t}+H_{t} u_{t}, \\
\gamma_{1} \mid Y_{0} & \sim N\left(a_{1 \mid 0}, P_{1 \mid 0}\right),
\end{aligned} \quad u_{t} \stackrel{i i d}{\sim} \mathcal{N}(0, I) .
$$

For simplicity we assume that $G_{t} H_{t}^{\prime}=0$ and we write the non-zero rows of $H_{t}$ as $M_{t}, G_{t} G_{t}^{\prime}=\Sigma_{t}$ and $H_{t} H_{t}^{\prime}=\Sigma_{\eta t}$. Throughout $c_{t}$ and $d_{t}$ are assumed known.

In the context of our paper we have mostly worked with the simplest of models were, putting $\beta=1$ and writing $r_{t}^{d}$ to denote daily returns computed as (8),

$$
\log \left(r_{t}^{d 2}+\text { const }\right)=h_{t}+\varepsilon_{t}, \quad \text { and } \quad h_{t+1}=\mu(1-\phi)+\phi h_{t}+\eta_{t}
$$

where we condition on the mixture $s_{t}$ such that

$$
\varepsilon_{t} \mid s_{t}=i \sim \mathcal{N}\left(m_{i}, v_{i}^{2}\right) \quad \text { and } \quad \eta_{t} \sim \mathcal{N}\left(0, \sigma_{\eta}^{2}\right)
$$

So this puts $y_{t}=\log \left(r_{t}^{d 2}+\right.$ const $), c_{t}=\mu_{i}, G_{t}=\left(\sigma_{i} 0\right), \gamma_{t}=h_{t}$ and $Z_{t}=1$. Likewise $d_{t}=\mu(1-\phi), T_{t}=\phi$ and $H_{t}=\left(0 \sigma_{\eta}\right)$. Finally, for a stationary initial condition, $a_{1 \mid 0}=\mu$ and $P_{1 \mid 0}=\sigma_{\eta}^{2} /\left(1-\phi^{2}\right)$. This means that $\varepsilon_{t}=G_{t} u_{t}, \eta_{t}=H_{t} u_{t}$ and $u_{t}$ is a bivariate standard normal.

The Kalman filter is run for $t=1, \ldots, n$,

$$
\begin{aligned}
\gamma_{t+1 \mid t} & =d_{t}+T_{t} \gamma_{t \mid t-1}+K_{t} v_{t}, \quad P_{t+1 \mid t}=T_{t} P_{t \mid t-1} L_{t}^{\prime}+\Sigma_{\eta t}, \quad v_{t}=y_{t}-Z_{t} \gamma_{t \mid t-1}-c_{t}, \\
F_{t} & =Z_{t} P_{t \mid t-1} Z_{t}^{\prime}+\Sigma_{t}, \quad K_{t}=T_{t} P_{t \mid t-1} Z_{t}^{\prime} F_{t}^{-1}, \quad L_{t}=c T_{t}-K_{t} Z_{t} .
\end{aligned}
$$

Here $\gamma_{t+1 \mid t}=\mathrm{E}\left(\gamma_{t+1} \mid y_{1}, \ldots, y_{t}\right)$ while $P_{t+1 \mid t}$ is the corresponding mean square error. More detailed discussion of the state space form and the Kalman filter is given in Harvey (1989).

The simulation signal smoother (de Jong and Shephard (1995)) draws from the multivariate normal posterior

$$
\left(c_{1}+Z_{1} \gamma_{1}, \ldots, c_{n}+Z_{n} \gamma_{n}\right) \mid y, \theta
$$

where $\theta$ denotes the parameters of the model. Setting $r_{n}=0$ and $N_{n}=0$, and writing $D_{t}=$ $F_{t}^{-1}+K_{t}^{\prime} N_{t} K_{t}, n_{t}=F_{t}^{-1} v_{t}-K_{t}^{\prime} r_{t}$, we run for $t=n, \ldots, 1$,

$$
\begin{aligned}
& C_{t}=\Sigma_{t}-\Sigma_{t} D_{t} \Sigma_{t}, \quad \kappa_{t} \sim \mathcal{N}\left(0, C_{t}\right), \\
& r_{t-1}=Z_{t}^{\prime} F_{t}^{-1} v_{t}+L_{t}^{\prime} r_{t}-V_{t}^{\prime} C_{t}^{-1} \kappa_{t}, \quad V_{t}=\Sigma_{t}\left(D_{t} Z_{t}-K_{t}^{\prime} N_{t} T_{t}\right), \\
& N_{t-1}=Z_{t}^{\prime} F_{t}^{-1} Z_{t}+L_{t}^{\prime} N_{t} L_{t}+V_{t}^{\prime} C_{t}^{-1} V_{t} .
\end{aligned}
$$


Then $y_{t}-\Sigma_{t} n_{t}-\kappa_{t}$ is a draw from the signal $c_{t}+Z_{t} \gamma_{t} \mid y, \theta, c_{t+1}+Z_{t+1} \gamma_{t+1}, \ldots, c_{n}+Z_{n} \gamma_{n}$.

The freeware package SSFPack, due to Koopman, Shephard, and Doornik (1996), provides easy to use functions which perform Kalman filtering and the simulation signal smoothing for an arbitrary state space model.

\subsection{Augmented state space}

Suppose that we write

$$
c_{t}=X_{t} \beta, \quad d_{t}=W_{t} \beta, \quad \beta \sim \mathcal{N}(0, \Lambda)
$$

where $\beta$ is independent of the $u_{t}$ process. Then we can estimate the states and the regression parameter $\beta$ at the same time using the augmented Kalman filter and simulation smoother. The first of these ideas is due to de Jong (1991), the second to de Jong and Shephard (1995).

The augmented Kalman filter adds two equations to the Kalman filter (22) which is run with $c_{t}=0, d_{t}=0$, additionally computing

$$
V_{t}^{a}=-Z_{t} \gamma_{t \mid t-1}^{a}-X_{t}, \quad \gamma_{t+1 \mid t}^{a}=W_{t}+T_{t} \gamma_{t \mid t-1}^{a}+K_{t} V_{t}^{a}
$$

where $\gamma_{1 \mid 0}^{a}=W_{1}$. Here $V_{t}^{a}$ is a $\operatorname{dim}\left(y_{t}\right) \times \operatorname{dim}(\beta)$ matrix. The augmented innovations $V_{t}^{a}$ are the innovations resulting from running $-X_{t}$ through the Kalman filter (22) with $d_{t}=W_{t}$. Hence we can compute the posterior of $\beta \mid y$ by looking at the weighted least squares regression of $v_{t}$ on $V_{t}^{a} \beta$ with prior information $\beta \sim \mathcal{N}(0, \Lambda)$ and variances $F_{t}$. If we set $S_{1}=\Lambda^{-1}$ and $s_{1}=0$ (the notation of $s_{t}$ is local to this discussion) then recursively calculating

$$
s_{t+1}=s_{t}+V_{t}^{a \prime} F_{t}^{-1} v_{t} \quad \text { and } \quad S_{t+1}=S_{t}+V_{t}^{a \prime} F_{t}^{-1} V_{t}^{a},
$$

we have that $\beta \mid y, \theta \sim \mathcal{N}\left(-S_{n}^{-1} s_{n}, S_{n}^{-1}\right)$, where $\theta$ now denotes the remaining parameters in the model.

The random regression effect $\beta$ can be analytically integrated out of the joint density of $y$ and $\beta$ (given $\theta$ ) as

$$
\begin{aligned}
f(y \mid \theta) & =\int f(y \mid \beta, \theta) \pi(\beta) d \beta=\frac{f(y \mid \beta=0, \theta) \pi(\beta=0)}{\pi(\beta=0 \mid y, \theta)} \\
& \propto \prod_{t=1}^{n} F_{t}^{-1 / 2} \exp \left(-\frac{1}{2} \sum_{t=1}^{n} v_{t}^{2} / F_{t}\right)|\Lambda|^{-1 / 2} \exp \left(\frac{1}{2} s_{n}^{\prime} S_{n}^{-1} s_{n}\right)\left|S_{n}^{-1}\right|^{1 / 2},
\end{aligned}
$$

using the terms from the augmented Kalman filter. This result is due to de Jong (1991).

If we draw from $b \sim \beta \mid y, \theta$ we can calculate a new set of innovations $v_{t}^{s}=v_{t}+V_{t}^{a} b$, which are the innovations from running the Kalman filter on a state space with known $\beta=b$. Hence we can use the simulation signal smoother which draws $c_{1}+Z_{1} \gamma_{1}, \ldots, c_{n}+Z_{n} \gamma_{n} \mid y, \beta=b, \theta$ using 
the simulation signal smoother (23) just by plugging in the $v_{t}^{s}$ instead of the $v_{t}$. By using both of these draws we are actually sampling directly from the distribution of

$$
\left(\beta, c_{1}+Z_{1} \gamma_{1}, \ldots, c_{n}+Z_{n} \gamma_{n}\right) \mid y, \theta
$$

\section{References}

Atkinson, A. C. (1986). Monte-Carlo tests of separate families of hypotheses. Unpublished paper: Imperial College, London.

Berzuini, C., N. G. Best, W. R. Gilks, and C. Larizza (1997). Dynamic graphical models and Markov chain Monte Carlo methods. J. Am. Statist. Assoc. 92. Forthcoming.

Bollerslev, T. (1987). A conditional heteroskedastic time series model for speculative prices and rates of return. Rev. Economics and Statistics 69, 542-47.

Bollerslev, T., R. F. Engle, and D. B. Nelson (1994). ARCH models. In R. F. Engle and D. McFadden (Eds.), The Handbook of Econometrics, Volume 4, pp. 2959-3038. NorthHolland.

Bowman, K. O. and L. R. Shenton (1975). Omnibus test contours for departures from normality based on $\sqrt{b_{1}}$ and $b_{2}$. Biometrika 62, 243-50.

Carter, C. K. and R. Kohn (1994). On Gibbs sampling for state space models. Biometrika 81, $541-53$.

Chesney, M. and L. O. Scott (1989). Pricing European options: a comparison of the modified Black-Scholes model and a random variance model. Journal of Financial and Qualitative Analysis 24, 267-84.

Chib, S. (1995). Marginal likelihood from Gibbs output. J. Am. Statist. Assoc. 90, 1313-21.

Chib, S. and E. Greenberg (1994). Bayes inference for regression models with ARMA(p,q) errors. J. Econometrics 64, 183-206.

Chib, S. and E. Greenberg (1995). Understanding the Metropolis-Hastings algorithm. The American Statistican 49, 327-35.

Chib, S. and E. Greenberg (1996). Markov chain Monte Carlo simulation methods in econometrics. Econometric Theory 12, 409-31.

Cox, D. R. (1991). Long-range dependence, non-linearity and time irreversibility. J. Time Series Analysis 12, 329-35.

de Jong, P. (1991). The diffuse Kalman filter. Ann. Statist. 19, 1073-1083. 
de Jong, P. (1998). The scan sampler. Biometrika 85. Forthcoming.

de Jong, P. and N. Shephard (1995). The simulation smoother for time series models. Biometrika 82, 339-50.

Diebold, F. X. and M. Nerlove (1989). The dynamics of exchange rate volatility: a multivariate latent factor ARCH model. J. Appl. Econometrics 4, 1-21.

Doornik, J. A. (1996). Ox: Object Oriented Matrix Programming, 1.10. London: Chapman \& Hall.

Engle, R. F. (1982). Autoregressive conditional heteroskedasticity with estimates of the variance of the United Kingdom inflation. Econometrica 50, 987-1007.

Escobar, M. and M. West (1995). Bayesian density estimation and inference using mixtures. J. Am. Statist. Assoc. 90, 577-88.

Fuller, W. A. (1996). Introduction to Time Series (2nd ed.). New York: John Wiley.

Gallant, A. R. and G. Tauchen (1996). Which moments to match. Econometric Theory 12, $657-81$.

Gelfand, A. and A. F. M. Smith (1990). Sampling-based approaches to calculating marginal densities. J. Am. Statist. Assoc. 85, 398-409.

Geman, S. and D. Geman (1984). Stochastic relaxation, Gibbs distribution and the Bayesian restoration of images. IEEE Transactions, PAMI 6, 721-41.

Geweke, J. (1994). Comment on bayesian analysis of stochastic volatility models. J. Business and Economic Statist. 12, 397-399.

Ghysels, E., A. C. Harvey, and E. Renault (1996). Stochastic volatility. In C. R. Rao and G. S. Maddala (Eds.), Statistical Methods in Finance, pp. 119-191. Amsterdam: North-Holland.

Gilks, W. K., S. Richardson, and D. J. Spiegelhalter (1996). Markov Chain Monte Carlo in Practice. London: Chapman \& Hall.

Gilks, W. R., N. G. Best, and K. K. C. Tan (1995). Adaptive rejection Metropolis sampling within Gibbs sampling. Applied Statistics 44, 155-73.

Gilks, W. R. and P. Wild (1992). Adaptive rejection sampling for Gibbs sampling. Applied Statistics 41, 337-348.

Gordon, N. J., D. J. Salmond, and A. F. M. Smith (1993). A novel approach to non-linear and non-Gaussian Bayesian state estimation. IEE-Proceedings F 140, 107-33. 
Gourieroux, C. and A. Monfort (1994). Testing non-nested hypotheses. In R. F. Engle and D. McFadden (Eds.), The Handbook of Econometrics, Volume 4, pp. 2583-637. NorthHolland.

Gourieroux, C., A. Monfort, and E. Renault (1993). Indirect inference. J. Appl. Econometrics 8, S85-S118.

Harvey, A. C. (1989). Forecasting, Structural Time Series Models and the Kalman Filter. Cambridge: Cambridge University Press.

Harvey, A. C., E. Ruiz, and N. Shephard (1994). Multivariate stochastic variance models. Rev. Economic Studies 61, 247-64.

Hastings, W. K. (1970). Monte-carlo sampling methods using markov chains and their applications. Biometrika 57, 97-109.

Hinde, J. (1992). Choosing between non-nested models: a simulation approach. In L. Fahrmeir, B. Francis, R. Gilchrist, and G. Tutz (Eds.), Advances in GLIM and Statistical Modelling, Proceedings of the GLIM92 Conference and the 7th International Workshop on Statistical Modelling, Munich, 13-17 July 1992, pp. 119-24. New York: Springer-Verlag.

Hull, J. and A. White (1987). The pricing of options on assets with stochastic volatilities. J. Finance 42, 281-300.

Isard, M. and A. Blake (1996). Contour tracking by stochastic propagation of conditional density. Proceedings of the European Conference on Computer Vision, Cambridge 1, 343356.

Jacquier, E., N. G. Polson, and P. E. Rossi (1994). Bayesian analysis of stochastic volatility models (with discussion). J. Business and Economic Statist. 12, 371-417.

Jacquier, E., N. G. Polson, and P. E. Rossi (1995). Models and prior distributions for multivariate stochastic volatility. Unpublished paper: Graduate School of Business, University of Chicago.

King, M., E. Sentana, and S. Wadhwani (1994). Volatility and links between national stock markets. Econometrica 62, 901-933.

Kitagawa, G. (1996). Monte Carlo filter and smoother for non-Gaussian nonlinear state space models. Journal of Computational and Graphical Statistics 5, 1-25.

Koopman, S. J., N. Shephard, and J. A. Doornik (1996). SSFPack 1.1: filtering, smoothing and simulation algorithms for state space models in Ox. Unpublished paper and software: available at http://www.nuff.ox.ac.uk/users/shephard/ox/. 
Mahieu, R. and P. Schotman (1997). Stochastic volatility and the distribution of exchange rate news. J. Appl. Econometrics 15. Forthcoming.

Marriott, J. M. and A. F. M. Smith (1992). Reparameterization aspects of numerical Bayesian methodology for autoregressivce moving-average models. J. Time Series Analysis 13, 32743.

Metropolis, N., A. W. Rosenbluth, M. N. Rosenbluth, A. H. Teller, and E. Teller (1953). Equations of state calculations by fast computing machines. J Chemical Physics 21, 108792.

Muller, P. (1991). Numerical integration in general dynamic models. Contemporary Mathematics 115, 145-63.

Park, S. and K. Miller (1988). Random number generators: good ones are hard to find. Communications of the ACM 31, 1192-201.

Pesaran, M. H. and B. Pesaran (1993). A simulation approach to the problem of computing Cox's statistic for testing nonnested models. J. Econometrics 57, 377-92.

Phillips, P. C. B. (1991). To criticise the critics: an objective Bayesian analysis of stochastic trends. J. Appl. Econometrics 6, 333-64.

Pitt, M. K. and N. Shephard (1997). Filtering via simulation: an auxiliary variable approach. working paper, Nuffield College, Oxford.

Pitt, M. K. and N. Shephard (1998). Analytic convergence rates and parameterisation issues for the Gibbs sampler applied to state space models. J. Time Series Analysis 19. Forthcoming.

Priestley, M. B. (1981). Spectral Analysis and Time Series. London: Academic Press.

Richardson, S. and P. Green (1997). On Bayesian analysis of mixtures with unknown number of components. J. R. Statist. Soc. B 59. Forthcoming.

Ripley, B. D. (1977). Modelling spatial patterns (with discussion). J. R. Statist. Soc. B 39, $172-212$.

Ripley, B. D. (1987). Stochastic Simulation. New York: Wiley.

Rosenblatt, M. (1952). Remarks on a multivariate transformation. Annals of Mathematical Statistics 23, 470-2.

Schotman, P. C. and H. K. Van Dijk (1991). A Bayesian analysis of the unit root in real exchange rates. J. Econometrics 49, 195-238. 
Shephard, N. (1993). Fitting non-linear time series models, with applications to stochastic variance models. J. Appl. Econometrics 8, S135-52.

Shephard, N. (1994). Partial non-Gaussian state space. Biometrika 81, 115-31.

Shephard, N. (1996). Statistical aspects of ARCH and stochastic volatility. In D. R. Cox, O. E. Barndorff-Nielson, and D. V. Hinkley (Eds.), Time Series Models in Econometrics, Finance and Other Fields, pp. 1-67. London: Chapman \& Hall.

Shephard, N. and S. Kim (1994). Comment on 'Bayesian analysis of stochastic volatility models' by Jacquier, Polson and Rossi. J. Business and Economic Statist. 11, 406-410.

Shephard, N. and M. K. Pitt (1997). Likelihood analysis of non-Gaussian measurement time series. Biometrika 84. Forthcoming.

Tanner, M. A. (1996). Tools for Statistical Inference: methods for exploration of posterior distributions and likelihood functions (3 ed.). New York: Springer-Verlag.

Tanner, M. A. and W. H. Wong (1987). The calculation of posterior distributions by data augmentation (with discussion). J. Am. Statist. Assoc. 82, 528-50.

Taylor, S. J. (1986). Modelling Financial Time Series. Chichester: John Wiley.

Taylor, S. J. (1994). Modelling stochastic volatility. Mathematical Finance 4, 183-204.

Tierney, L. (1994). Markov Chains for exploring posterior distributions (with discussion). Ann. Statist. 21, 1701-62.

Titterington, D. M., A. F. M. Smith, and U. E. Makov (1985). Statistical Analysis of Finite Mixture Distributions. Chichester: Wiley.

West, M. (1993). Approximating posterior distributions by mixtures. J. R. Statist. Soc. B 55, $409-42$. 